\title{
High-accuracy numerical simulation of black-hole binaries: Computation of the gravitational- wave energy flux and comparisons with post-Newtonian approximants
}

\author{
Michael Boyle, ${ }^{1}$ Alessandra Buonanno, ${ }^{2}$ Lawrence E. Kidder, ${ }^{3}$ Abdul H. Mroué, ${ }^{3}$ Yi Pan, ${ }^{2}$ \\ Harald P. Pfeiffer, ${ }^{1}$ and Mark A. Scheel ${ }^{1}$ \\ ${ }^{1}$ Theoretical Astrophysics 130-33, California Institute of Technology, Pasadena, California 91125, USA \\ ${ }^{2}$ Maryland Center for Fundamental Physics, Department of Physics, University of Maryland, College Park, Maryland 20742, USA \\ ${ }^{3}$ Center for Radiophysics and Space Research, Cornell University, Ithaca, New York, 14853, USA
}

(Received 25 April 2008; published 18 November 2008)

\begin{abstract}
Expressions for the gravitational-wave (GW) energy flux and center-of-mass energy of a compact binary are integral building blocks of post-Newtonian (PN) waveforms. In this paper, we compute the GW energy flux and GW frequency derivative from a highly accurate numerical simulation of an equal-mass, nonspinning black-hole binary. We also estimate the (derivative of the) center-of-mass energy from the simulation by assuming energy balance. We compare these quantities with the predictions of various PN approximants [adiabatic Taylor and Padé models; nonadiabatic effective-one-body (EOB) models]. We find that Padé summation of the energy flux does not accelerate the convergence of the flux series; nevertheless, the Padé flux is markedly closer to the numerical result for the whole range of the simulation (about $30 \mathrm{GW}$ cycles). Taylor and Padé models overestimate the increase in flux and frequency derivative close to merger, whereas EOB models reproduce more faithfully the shape of and are closer to the numerical flux, frequency derivative, and derivative of energy. We also compare the GW phase of the numerical simulation with Padé and EOB models. Matching numerical and untuned 3.5 PN order waveforms, we find that the phase difference accumulated until $M \omega=0.1$ is -0.12 radians for Padé approximants, and 0.50 (0.45) radians for an EOB approximant with Keplerian (non-Keplerian) flux. We fit free parameters within the EOB models to minimize the phase difference, and confirm the presence of degeneracies among these parameters. By tuning the pseudo 4PN order coefficients in the radial potential or in the flux, or, if present, the location of the pole in the flux, we find that the accumulated phase difference at $M \omega=0.1$ can be reduced-if desired-to much less than the estimated numerical phase error (0.02 radians).
\end{abstract}

DOI: 10.1103/PhysRevD.78.104020

PACS numbers: 04.25.D-, 04.25.dg, 04.25.Nx, 04.30.-w

\section{INTRODUCTION}

The first-generation interferometric gravitational-wave (GW) detectors, such as LIGO [1,2], GEO600 [3], and Virgo [4,5], are now operating at or near their design sensitivities. One of the most promising sources for these detectors is the inspiral and merger of binary black holes (BBHs) with masses $m_{1} \sim m_{2} \sim 10-20 M_{\odot}$ [6,7]. A detailed and accurate understanding of the gravitational waves radiated as the black holes spiral towards each other will be crucial not only for the initial detection of such sources, but also for maximizing the information that can be obtained from signals once they are observed. Both the detection and subsequent analysis of gravitational waves from compact binaries depends crucially on our ability to build an accurate bank of templates, where each template is a theoretical model that accurately represents the gravitational waveform from a binary that has a certain set of parameters (e.g., masses and spins). For detection, the technique of matched filtering is applied to noisy data to extract any signals that match members of the template bank. For analysis, the best-fit parameters are determined, most likely by an iterative process that involves constructing further templates to zero in on the best fit.
When the black holes are far apart and moving slowly, the gravitational waveform (i.e., the template) can be accurately computed using a post-Newtonian (PN) expansion. As the holes approach each other and their velocities increase, the post-Newtonian expansion is expected to become less and less reliable. However, until recently there has been no independent way to determine how close comparable-mass holes must be before PN methods become inaccurate. This has changed with recent advances in numerical relativity (NR), which make it possible for the first time to quantify the disagreement between PN predictions [8] and the true waveform [9-14]. In a previous paper [12], some of us described numerical simulations of 15 orbits of an equal-mass, nonspinning binary black-hole system. Gravitational waveforms from these simulations covering more than $30 \mathrm{GW}$ cycles and ending about 1.5 GW cycles before merger, were compared with those from quasicircular PN formulas for several time-domain Taylor approximants computed in the so-called adiabatic approximation. We found that there was excellent agreement (within 0.05 radians) in the GW phase between the numerical results and the PN waveforms over the first $\sim 15$ cycles, thus validating the numerical simulation and establishing a regime where PN theory is accurate. In the last 15 
cycles to merger, however, generic time-domain Taylor approximants build up phase differences of several radians. But, apparently by coincidence, one specific PN approximant, TaylorT4 at 3.5PN order, agreed much better with the numerical simulations, with accumulated phase differences of less than 0.05 radians over the 30-cycle waveform. Simulations by Hannam et al. [13] for equal-mass, nonprecessing spinning binaries confirm that this agreement in the nonspinning case is a coincidence: they find the phase disagreement between TaylorT4 and the numerical waveform can be a radian or more as the spins of the black holes are increased.

To build a template bank to be used by ground-based GW detectors, one possibility would be to run a separate numerical simulation for each template. This is not currently possible, however, due to the large computational cost per numerical waveform (on the order of a week for a single waveform) and the large number of templates needed to cover the parameter space, especially when spins are present. A more realistic possibility is to perform a small number of simulations and develop an analytic template family (i.e., a fitting formula) which interpolates the parameter space between the simulations [15-20].

Before the NR breakthrough several analytic prescriptions were proposed to address the loss of accuracy of the adiabatic Taylor approximants. Damour, Iyer, and Sathyaprakash [21] introduced the Padé summation of the PN center-of-mass energy and gravitational energy flux in order to produce a series of Padé approximants for the waveforms in the adiabatic approximation. Buonanno and Damour [22-25] introduced the effectiveone-body (EOB) approach which gives an analytic description of the motion and radiation beyond the adiabatic approximation of the binary system through inspiral, merger, and ringdown. The EOB approach also employs the Padé summation of the energy flux and of some crucial ingredients, such as the radial potential entering the conservative dynamics. So far, the EOB waveforms have been compared with several numerical waveforms of nonspinning binary black holes $[9,15,16,18-20]$. Buonanno et al. [16] showed that by using three quasinormal modes [9] and by tuning the pseudo 4PN order coefficient [26] in the EOB radial potential to a specific value, the phase difference accumulated by the end of the ringdown phase can be reduced to $\sim 0.19-0.50$ radians, depending on the mass ratio and the number of multipole moments included in the waveform. Those results were obtained using waveforms with 5-16 GW cycles and mass ratios 1:4, 1:2, 2:3, and 1:1. In Refs. [18-20], the authors introduced other improvements in the EOB approach, in part obtained by tuning the test-mass limit results [27]—for example, Padé summation of the PN amplitude corrections in the inspiral waveform; ringdown matching over an interval instead of a point; inclusion of noncircular terms in the tangential damping force; use of five quasinormal modes. They found that the phase differences accumulated by the end of the inspiral (ringdown) can be reduced to \pm 0.001 ( \pm 0.03 ) radians for equal-mass binaries $[18,19]$ and to \pm 0.05 radians for binaries with mass ratio 1:2 [20]. Note that these phase differences are smaller than the numerical errors in the simulations.

The energy flux and the center-of-mass energy are two fundamental quantities of the binary dynamics and crucial ingredients in building GW templates. In this paper, we extract these quantities, and compare the results from our numerical inspiral simulation [12] with PN results in both their Taylor-expanded and summed (Padé and EOB) forms. The agreement between the numerical and analytical results at large separation for the energy flux and the centerof-mass energy is a further validation of the numerical simulation. It also allows us to study whether or not the agreement of the phase evolution of PN and numerical waveforms is accidental. In addition, we compute waveforms based on adiabatic Padé and nonadiabatic EOB approximants in their untuned form (i.e., without introducing fitting coefficients) and study their agreement with our numerical simulations.

We try to understand whether these approximants can reproduce features of the numerical simulations that can be exploited to develop a faithful analytic template family. By introducing unknown higher-order PN coefficients into the dynamics and tuning them to the numerical data, we investigate how to improve the agreement with the numerical results. Although our study only examines nonspinning, equal-mass binary black holes, by combining it with other studies [15-20] one can already pinpoint which parameters are degenerate and which have the largest effect on the waveforms. This is particularly relevant during the last stages of inspiral and plunge. The overall methodology can be extended to a larger region of the parameter space. We will defer to a future paper a complete study of the flexibility of the EOB approach with the extension of our numerical waveform through merger and ringdown.

This paper is organized as follows: Sec. II gives a quick review of the numerical simulations presented in [12], and then presents the computation of the GW energy flux from the simulation. In Sec. III, we summarize the PN approximants that will be compared to the numerical simulation. In Sec. IV, we compare the GW energy flux for the various PN approximants with numerical results and explore the possibility of improving the agreement with the numerical flux by adding phenomenological parameters $[15,16,18-$ 20]. In Sec. V, we examine the evolution of the center-ofmass energy for the various PN approximants and compare to the numerical results assuming balance between the change in the center-of-mass energy and the energy carried from the system by the gravitational waves. In Sec. VI, we compare waveforms constructed from the Padé and EOB approximants with our numerical results, and study how to improve the agreement by exploiting the flexibility of the 
EOB model (i.e., by fitting free parameters of the EOB model). Finally, we present some concluding remarks in Sec. VII. In the Appendix we review the performance of the Padé summation of the Taylor series of the energy flux in the test particle limit.

\section{COMPUTATION OF THE NUMERICAL GRAVITATIONAL-WAVE ENERGY FLUX}

\section{A. Overview and definitions}

The data used in this paper is the same as that described in Sec. II of Boyle et al. [12]. The simulation is a 16-orbit inspiral, with very low spin and eccentricity. Figure 1 presents a view of some relevant quantities of that simulation.

The Newman-Penrose scalar $\Psi_{4}$, defined using a coordinate-based tetrad, is extracted from the simulation at several extraction radii and expanded in spin-weighted spherical harmonics,

$$
\Psi_{4}(t, r, \theta, \phi)=\sum_{l, m} \Psi_{4}^{l m}(t, r)_{-2} Y_{l m}(\theta, \phi) .
$$

Then $\Psi_{4}^{l m}(t, r)$ is extrapolated to infinite extraction radius using an $n$-th order polynomial in $1 / r$, where typically $n=$ 3 . This results in the asymptotic field $r \Psi_{4}^{l m}\left(t-r^{*}\right)$ as function of retarded time ${ }^{1} t-r^{*}$.

Gravitational radiation may also be expressed via the standard metric-perturbation quantities $h_{+}$and $h_{\times}$, which we similarly write in terms of spin-weighted spherical harmonic components,

$$
h \equiv h_{+}-i h_{\times}=\sum_{l, m} h_{l m-2} Y_{l m} .
$$

For linear perturbations around Minkowski space, $\Psi_{4}^{l m}(t-$ $\left.r^{*}\right)=\ddot{h}_{l m}\left(t-r^{*}\right)$. In particular, this relation should be true for the waveforms we have extrapolated to infinity.

However, to compute the energy flux we do not need to determine $h$; we need only its time derivative $\dot{h}$. The energy flux depends on the spin-weighted spherical harmonic coefficients of the time derivative $\dot{h}$ via

$$
F=\frac{1}{16 \pi} \sum_{l=2}^{\infty} \sum_{m=-l}^{l}\left|r \dot{h}_{l m}\right|^{2}
$$

We obtain $\dot{h}_{l m}$ by time-integration of $\Psi_{4}^{l m}$, as discussed in detail below.

Finally, we define gravitational-wave phase and frequency in two ways-one based on $\Psi_{4}^{22}$, and one based on $\dot{h}_{22}$ :

$$
\phi=-\arg \left(\Psi_{4}^{22}\right), \quad \omega=\frac{d}{d t} \phi,
$$

\footnotetext{
${ }^{1}$ See Sec. II F of Ref. [12] for a precise definition of $r^{*}$ and a description of the extrapolation.
}

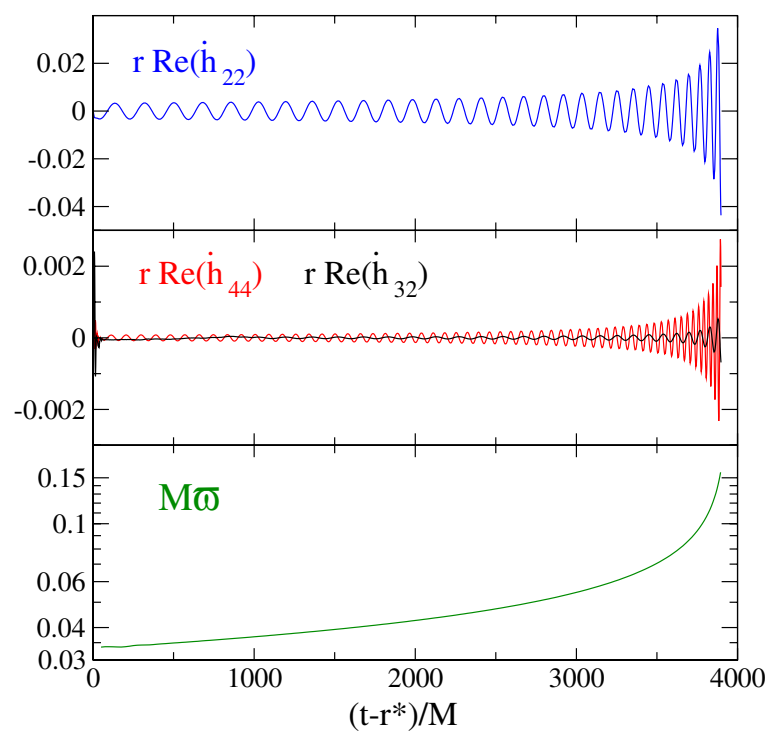

FIG. 1 (color online). Some aspects of the numerical simulation. From top panel to bottom: the leading mode $\dot{h}_{22}$; the two next largest modes, $\dot{h}_{44}$ and $\dot{h}_{32}$ (smallest); the frequency of $\dot{h}_{22}$ [see Eq. (5)].

$$
\varphi=-\arg \left(\dot{h}_{22}\right), \quad \varpi=\frac{d}{d t} \varphi .
$$

In both cases, we define the arg function to be the usual function, with discontinuities of $2 \pi$ removed. Many PN formulae (see Sec. III) involve yet another frequency and phase: the orbital phase $\Phi$ and orbital frequency $\Omega$. Although the three frequencies satisfy $\omega \approx \varpi \approx 2 \Omega$, the slight differences between different frequencies are significant at the level of precision of our comparison (see Fig. 6 below), so it is important to distinguish carefully between them.

When discussing our numerical solution, we write all dimensionful quantities in terms of the mass scale $M$, which we choose to be the sum of the irreducible masses of the two black holes. ${ }^{2}$

\section{B. Calculation of $\dot{h}$}

The energy flux depends on the spin-weighted spherical harmonic coefficients of $\dot{h}$ via Eq. (3). We therefore need to perform one time integration on $\Psi_{4}^{l m}$ :

$$
\dot{h}_{l m}(t)=\int_{t_{0}}^{t} \Psi_{4}^{l m}\left(t^{\prime}\right) d t^{\prime}+H_{l m} .
$$

This integration is performed for each mode $(l, m)$ separately and requires the choice of two integration constants, which are contained in the complex number $H_{l m}$. Ideally, $H_{l m}$ should be chosen such that $\dot{h}_{l m} \rightarrow 0$ for $t \rightarrow-\infty$. Because our numerical simulations do not extend into the distant past, this prescription cannot be implemented.

\footnotetext{
${ }^{2}$ This quantity was denoted by $m$ in Ref. [12].
} 
Rather, we make use of the approximation that the real and imaginary parts of $\dot{h}_{l m}$ should oscillate symmetrically around zero.

Let us consider a pure sine/cosine wave, with constant amplitude and phase:

$$
\begin{gathered}
\Psi_{4}^{\mathrm{ex}}=A[\cos (\omega t)+i \sin (\omega t)], \\
\dot{h}^{\mathrm{ex}}=\frac{A}{\omega}[\sin (\omega t)-i \cos (\omega t)]+H^{\mathrm{ex}},
\end{gathered}
$$

where the superscript "ex" stands for example. The amplitude is given by

$$
\begin{aligned}
\left|\dot{h}^{\mathrm{ex}}\right|^{2}= & \frac{A^{2}}{\omega^{2}}+2 \frac{A}{\omega}\left[\operatorname{Re} H^{\mathrm{ex}} \sin (\omega t)-\operatorname{Im} H^{\mathrm{ex}} \cos (\omega t)\right] \\
& +\left|H^{\mathrm{ex}}\right|^{2} .
\end{aligned}
$$

Only for the correct choice of integration constants, $H^{\mathrm{ex}}=$ 0 , is the amplitude $\left|\dot{h}^{\mathrm{ex}}\right|$ constant.

Therefore, we propose to determine the integration constants $H_{l m}$ in Eq. (6) by minimizing the time derivative of the amplitude over a long part of the waveform. In particular, we minimize

$$
I_{l m} \equiv \int_{t_{1}}^{t_{2}}\left(\frac{d}{d t}\left|\dot{h}_{l m}\right|^{2}\right)^{2} d t .
$$

From this minimization principle it follows that $H_{l m}$ is determined by the linear system

$$
\begin{aligned}
\operatorname{Re} H & \int\left(\operatorname{Re} \Psi_{4}\right)^{2} d t+\operatorname{Im} H \int \operatorname{Re} \Psi_{4} \operatorname{Im} \Psi_{4} d t \\
& =-\int\left[\left(\operatorname{Re} \Psi_{4}\right)^{2} \operatorname{Re} \dot{h}_{0}+\operatorname{Re} \Psi_{4} \operatorname{Im} \Psi_{4} \operatorname{Im} \dot{h}_{0}\right] d t \\
\operatorname{Re} H & \int \operatorname{Re} \Psi_{4} \operatorname{Im} \Psi_{4} d t+\operatorname{Im} H \int\left(\operatorname{Im} \Psi_{4}\right)^{2} d t \\
& =-\int\left[\left(\operatorname{Im} \Psi_{4}\right)^{2} \operatorname{Im} \dot{h}_{0}+\operatorname{Re} \Psi_{4} \operatorname{Im} \Psi_{4} \operatorname{Re} \dot{h}_{0}\right] d t .
\end{aligned}
$$

Here, we have suppressed the indices $\operatorname{lm}$ for clarity, all integrals are definite integrals from $t_{1}$ to $t_{2}$, and $\dot{h}_{0}(t) \equiv$ $\int_{t_{0}}^{t} \Psi_{4}\left(t^{\prime}\right) d t^{\prime}$. For a given integration interval $\left[t_{1}, t_{2}\right]$, Eqs. (11) provide a deterministic procedure to determine the integration constants $H_{l m}$. We note that there have been several earlier proposals to fix integration constants [20,28-31]. While we have not tested those proposals, we point out that Eqs. (11) allow for very accurate determination of the integration constants and one can easily obtain an error estimate, as we discuss in the next subsection.

\section{Uncertainties in numerical quantities}

Because the amplitude and frequency of the waveform are not constant, this procedure is imperfect, and the result depends somewhat on the chosen values of $t_{1}$ and $t_{2}$. To estimate the residual uncertainty in $H$ due to this choice, we select nine different values for $t_{1}$ and 11 values for $t_{2}$ : $t_{1}=200 M, 220 M, \ldots, 360 M ; \quad t_{2}=2000 M, 2100 M, \ldots$, $3000 \mathrm{M}$. The values of $t_{1}$ vary over roughly one GW cycle and test the sensitivity to the GW phase at the start of the integration interval; the values of $t_{2}$ are designed to test the dependence on the amplitude at the end of the integration interval. For $t_{2}>3000 \mathrm{M}$ we find that the errors in our procedure rapidly increase for several reasons: (a) the minimization principle is based on the approximation that the amplitude is constant; this approximation becomes worse toward merger; (b) $I_{l m}$ in Eq. (10) weights absolute changes in $|\dot{h}|$, not relative ones; close to merger, the amplitude becomes so large that it dominates $I_{l m}$; and (c) the integration constants shift the waveform $\dot{h}_{l m}$ vertically, and we are trying to determine the particular vertical shift such that $\dot{h}_{l m}$ is centered around zero. Determination of such an offset is most accurate in a regime where the oscillations are small, i.e., at early times.

For each of these 99 integration intervals, we compute integration constants using Eqs. (11) for the three dominant modes, $\dot{h}_{22}, \dot{h}_{44}$, and $\dot{h}_{32}$; we compute $F(t)$ from Eq. (3) using only these modes; and we compute $\varpi(t)$. (We will show below that the contributions of other modes are far below our numerical errors on the flux.) We average the 99 functions $F(t)$ and $\varpi(t)$, and then use a parametric plot of $F(t)$ versus $\varpi(t)$ in our comparisons presented below. The variation in these 99 values yields an uncertainty in $F$ due to the choice of integration constants.

The lower panel of Fig. 2 shows the variation in flux from the 99 different integration intervals. We find that the maximum deviation can be well approximated by $\max |\delta F| / F=1.5 \times 10^{-5}(M \varpi)^{-3 / 2}$ (see the solid line in the lower panel of Fig. 2). The average $F$ computed from all 99 intervals $\left[t_{1}, t_{2}\right]$ will have a smaller error. Inspection of the lower panel of Fig. 2 reveals that the $\delta F / F$ curves fall into 11 groups, corresponding to the 11 values of $t_{2}$. Assuming that $\delta F$ between these groups is randomly distributed, the error of the average will be reduced by a factor $\sqrt{11}$, i.e., $\delta F / F=5 \times 10^{-6}(M \varpi)^{-3 / 2}$. This error is indicated as the gray shaded area in the upper panel of Fig. 2.

The upper panel of Fig. 2 plots the relative change in $F(\varpi)$ for several changes in our numerical simulation: (a) computing the flux from a run with lower resolution (30c-1/N5 in the language of Boyle et al. [12]); (b) using a different set of extraction radii for the extraction of the gravitational wave; (c) increasing the polynomial order of extrapolation of $\Psi_{4}$ to infinite extraction radius from $n=3$ to $n=4$; and (d) computing the flux from a separate evolution with a different outer boundary radius (30c-2/N6). At low frequencies, the error is dominated by extrapolation to infinite radius and is a few tenths of a percent; at intermediate frequencies, $0.055 \lesssim M \varpi<$ 0.083 , all errors are smaller than $0.1 \%$. At frequency $M \varpi \approx 0.084$ we change the gauge conditions in the evolutions to allow wave escorting; this introduces high- 


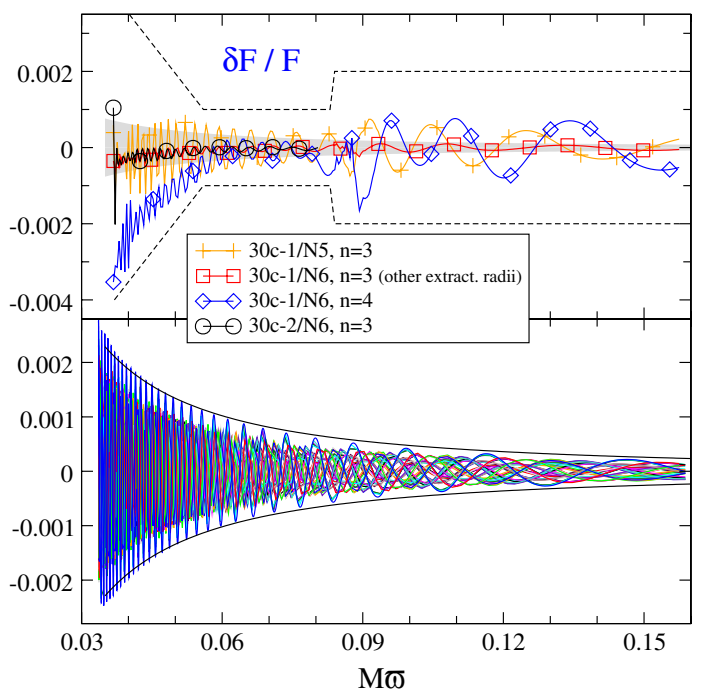

FIG. 2 (color online). Lower panel: relative difference between flux $F(\varpi)$ computed with 99 different intervals $\left[t_{1}, t_{2}\right]$ and the average of these. Upper panel: relative change in the flux $F(\varpi)$ under various changes to the numerical simulation. The gray area in the upper panel indicates the uncertainty due to the choice of integration constants, which is always dominated by numerical error. The dashed line in the upper panel is our final error estimate, which we plot in later figures.

frequency features, which are small when extrapolation order $n=3$ is used, but which dominate for $n=4$ extrapolation. The numerical data we use in the PN comparisons below is extrapolated with $n=3$, for which the features due to change of gauge are small, but nevertheless we will use conservative error bars encompassing the $n=$ 4 extrapolation as indicated in Fig. 2, i.e. a relative error of $0.2 \%$ for $M \varpi>0.083$. We find that the uncertainty in the flux due to numerical error in determining $\Psi_{4}$ is always larger than the uncertainty due to the choice of integration constants.

The contributions of the various $(l, m)$ modes to the total flux [see Eq. (3)] are plotted in Fig. 3. The top panel plots the flux as a function of time; the lower panel as a function of frequency $M \varpi$. The dashed line in the lower panel corresponds to the error estimate of Fig. 2. Because the modes $(5,4),(6,6)$, and $(8,8)$ are significantly smaller than our error estimate, we do not include them in the present analysis.

To estimate the uncertainty in $\dot{\varpi}$, we proceed in a similar fashion. Each one of the 99 different integration intervals yields an $\dot{h}_{22}$ from which we determine $\dot{\varpi}$. We average these to obtain the final $\dot{\varpi}$ to be used in the post-Newtonian comparisons. The lower panel of Fig. 4 shows the variation in $\dot{\varpi}$ between the 99 different integration intervals. We find that the maximum deviation can be well approximated by $\max \left|M^{2} \delta \dot{\varpi}\right|=5 \times 10^{-6}(M \varpi)^{-0.3}$ (see the solid line in lower panel of Fig. 4). The average $\dot{\varpi}$ computed from all 99 intervals $\left[t_{1}, t_{2}\right]$ will have a smaller error. Inspection of
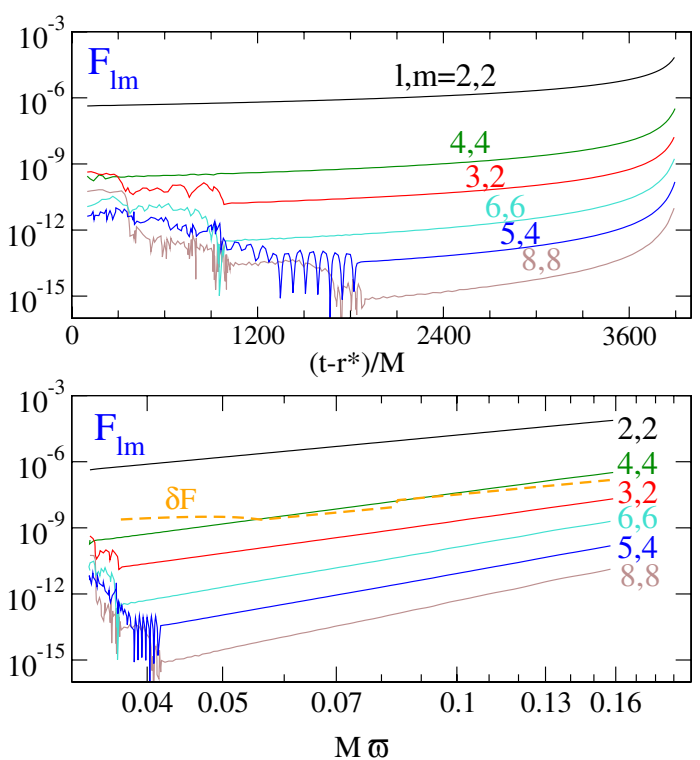

FIG. 3 (color online). Contributions of various $(l, m)$ modes to the total numerical gravitational-wave flux. Upper panel: plotted as a function of time. Lower panel: plotted as a function of frequency $M \varpi$. The lower panel also contains the error estimate derived in Fig. 2.

the lower panel of Fig. 4 reveals that the $\delta \dot{\varpi}$ curves fall into 11 groups, corresponding to the 11 values of $t_{2}$. As for the case of $\delta F$, if we assume that $\delta \dot{\varpi}$ between these groups is randomly distributed, then the error of the average will be

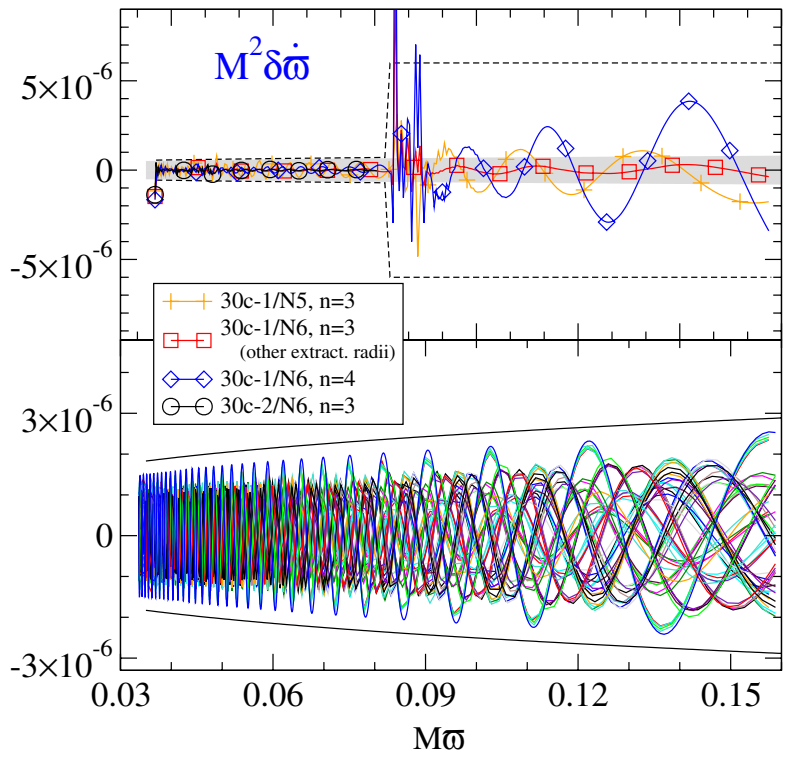

FIG. 4 (color online). Lower panel: difference between frequency derivative $\dot{\varpi}$ computed with 99 different intervals $\left[t_{1}, t_{2}\right]$ and the average of these. Upper panel: change in the frequency derivative $\dot{\varpi}$ under various changes to the numerical simulation. The gray area in the upper panel indicates the uncertainty due to choice of integration constants, which dominates the overall uncertainty for low frequencies. The dashed line in the upper panel is our final error estimate, which we plot in later figures. 
reduced by a factor $\sqrt{11}$, i.e., $M^{2} \delta \dot{\varpi}=1.5 \times$ $10^{-6}(M \varpi)^{-0.3}$. This error is indicated as the gray shaded area in the upper panel of Fig. 4.

The upper panel of Fig. 4 plots also the change in $\dot{\varpi}(\varpi)$ for the same changes in our numerical simulation already discussed above. We find that at $M \varpi<0.083$, the uncertainty in $\dot{\varpi}$ is dominated by the choice of integration constants, whereas at higher frequencies the uncertainty is dominated by the numerical errors in the calculation of $\Psi_{4}$. As discussed above, at frequency $M \varpi \approx 0.084$ we change the gauge conditions in the evolutions to allow wave escorting; this introduces high-frequency features leading to more conservative error estimates.

Note that $\dot{\varpi}$ is a very steep function of $\varpi$. While the absolute errors in $\dot{\varpi}$ are roughly constant for our simulation, the relative errors change significantly: $\delta \dot{\varpi} / \dot{\varpi}$ drops from about $10 \%$ early in the run to about $0.2 \%$ at late times.

We also point out that the first $1000 \mathrm{M}$ of our simulation are contaminated by noise due to a pulse of "junkradiation" at the start of the simulation. While this contamination is not apparent on a plot of the waveform as in Fig. 1, it nevertheless limits accurate PN-NR comparisons to the region, $t-r^{*} \gtrsim 1000 M$, i.e., $M \varpi \gtrsim 0.037$.

\section{POST-NEWTONIAN APPROXIMANTS}

In this paper, we will compare the numerical simulation to various approximants based on the PN expansion. The PN expansion is a slow-motion, weak-field approximation to general relativity with an expansion parameter $\epsilon \sim$ $(v / c)^{2} \sim\left(G M / r c^{2}\right)$. For a binary system of two point masses $m_{1}$ and $m_{2}, v$ is the magnitude of the relative velocity, $M$ is the total mass, and $r$ is the separation. For a review of the PN expansion applied to gravitational radiation from inspiralling compact binaries, see Ref. [8].

In Table I we summarize the PN approximants that we use, and our notation. We shall use the PN approximants in the so-called adiabatic approximation, both in the standard Taylor-expanded form (reviewed in Sec. III A) and in a form based on Padé summation (reviewed in Sec. III B). In addition, we shall use the nonadiabatic EOB model (reviewed in Sec. III C) in its original form [22-24], as well as several variations that differ in the form of the radiationreaction force [32-34]. After summarizing the various PN approximants in Secs. III A, III B, and III C, we describe how we construct the waveform for these approximants in Sec. III D.

In the adiabatic approximation, the inspiral is modeled as a quasistationary sequence of circular orbits. The evolution of the inspiral (and, in particular, of the orbital phase $\Phi)$ is completely determined by the energy-balance equation [8]

$$
\frac{d E\left(v_{\Omega}\right)}{d t}=-F\left(v_{\Omega}\right)
$$

This equation relates the time derivative of the center-of-
TABLE I. Summary of PN approximants. The T approximants are always Taylor T4 [12] except in Fig. 16. The P approximant in the second row was introduced in Refs. [21,24,32] and the original $\mathrm{E}$ approximant in third row was introduced in Refs. [2224]. The last three rows refer to three possible variations of $\mathrm{E}$ approximants introduced in Refs. [32,33]. In a few tests aimed at improving the closeness between numerical data and $\mathrm{E}$ approximants, we vary $v_{\text {pole }}$ and treat the logarithms as constants when Padé summation to the flux is applied [18]. We shall denote this flux by $\bar{F}_{n}^{m}$. Finally, when using tuned PN approximants with pseudo 4PN order terms in the flux, energy, or Hamiltonian, we denote the latter as $p F, p E$, and $p H$. Note that if known testmass limit coefficients in the flux are used, the latter is still denoted as $F$ even at PN orders larger than 3.5PN. Finally, the values of $v_{\text {pole }}$ and $v_{\text {lso }}$ used in the P approximants $F_{n}^{m}$ and ${ }^{\mathrm{nK}} F_{n}^{m}$ are $v_{\mathrm{pole}}^{2 \mathrm{PN}}=0.6907$ and $v_{\mathrm{lso}}^{2 \mathrm{PN}}=0.4456$.

\begin{tabular}{lcccc}
\hline \hline Approximant & Notation & See Eqs. & Adiabatic & Keplerian \\
\hline Taylor (T) & $F_{n} / E_{p}$ & $(19)$ and (14) & yes & yes \\
Padé (P) & $F_{n}^{m} / E_{p}^{q}$ & $(39)$ and (33) & yes & yes \\
EOB (E) & $F_{n}^{m} / H_{p}$ & $(64)$ and (44) & no & yes \\
EOB (E) & ${ }^{n K} F_{n}^{m} / H_{p}$ & (65) and (44) & no & no \\
EOB (E) & $F_{n} / H_{p}$ & (69) and (44) & no & yes \\
EOB (E) & ${ }^{\mathrm{nK}} F_{n} / H_{p}$ & (70) and (44) & no & no \\
\hline \hline
\end{tabular}

mass energy $E\left(v_{\Omega}\right)$ (which is conserved in absence of radiation reaction) to the gravitational-wave energy flux $F\left(v_{\Omega}\right)$. Both functions are known for quasicircular orbits as a PN expansion in the invariantly defined velocity

$$
v_{\Omega}=(M \Omega)^{1 / 3},
$$

where $\Omega=\dot{\Phi}$ is the orbital frequency (we use units such that $G=c=1){ }^{3}$ We will denote the Taylor-expanded flux (energy) by $F_{k}\left(E_{k}\right)$ where $k$ denotes the maximum power of $v_{\Omega}$ retained in the series. (Recall that $k=2 N$ for an $N$ th order PN expansion.) We will denote the Padé-expanded flux (energy) by $F_{n}^{m}\left(E_{n}^{m}\right)$ where $m+n=k$, with $m$ and $n$ denoting the order of the polynomial in the numerator and denominator, respectively.

\section{A. Adiabatic Taylor approximants}

For generic values of the symmetric mass ratio $\nu=$ $m_{1} m_{2} / M^{2}$, the center-of-mass energy is known through 3PN order [35-39]. For circular orbits the Taylor PN approximants (henceforth, T approximants) to the energy are given by

$$
E_{2 k}\left(v_{\Omega}\right)=-\frac{M \nu}{2} v_{\Omega}^{2} \sum_{i=0}^{k} \mathcal{E}_{2 i}(\nu) v_{\Omega}^{2 i}
$$

where the known coefficients are

$$
\mathcal{E}_{0}(\nu)=1,
$$

\footnotetext{
${ }^{3}$ In Ref. [12], we used $x=v_{\Omega}^{2}$ as the expansion parameter.
} 


$$
\begin{gathered}
\mathcal{E}_{2}(\nu)=-\frac{3}{4}-\frac{\nu}{12}, \\
\mathcal{E}_{4}(\nu)=-\frac{27}{8}+\frac{19}{8} \nu-\frac{1}{24} \nu^{2}, \\
\mathcal{E}_{6}(\nu)=-\frac{675}{64}+\left(\frac{34445}{576}-\frac{205}{96} \pi^{2}\right) \nu-\frac{155}{96} \nu^{2} \\
-\frac{35}{5184} \nu^{3} .
\end{gathered}
$$

The GW energy flux for arbitrary masses has been computed through $3.5 \mathrm{PN}$ order [40-43]:

$$
F_{k}\left(v_{\Omega}\right)=\frac{32}{5} \nu^{2} v_{\Omega}^{10} \sum_{i=0}^{k} \mathcal{F}_{i}(\nu) v_{\Omega}^{i}
$$

where

$$
\begin{gathered}
\mathcal{F}_{0}(\nu)=1, \\
\mathcal{F}_{1}(\nu)=0 \\
\mathcal{F}_{2}(\nu)=-\frac{1247}{336}-\frac{35}{12} \nu, \\
\mathcal{F}_{3}(\nu)=4 \pi \\
\mathcal{F}_{4}(\nu)=-\frac{44711}{9072}+\frac{9271}{504} \nu+\frac{65}{18} \nu^{2}, \\
\mathcal{F}_{5}(\nu)=-\left(\frac{8191}{672}+\frac{583}{24} \nu\right) \pi, \\
\mathcal{F}_{6}(\nu)=\frac{6643739519}{69854400}+\frac{16}{3} \pi^{2}-\frac{1712}{105} \gamma_{E}-\frac{856}{105} \\
\times \log \left(16 v_{\Omega}^{2}\right)+\left(-\frac{134543}{7776}+\frac{41}{48} \pi^{2}\right) \nu \\
-\frac{94403}{3024} \nu^{2}-\frac{775}{324} \nu^{3}, \\
\mathcal{F}_{7}(\nu)=\left(-\frac{16285}{504}+\frac{214745}{1728} \nu+\frac{193385}{3024} \nu^{2}\right) \pi,
\end{gathered}
$$

where $\gamma_{E}$ is Euler's constant. Notice that starting at 3PN order $(k=6)$ logarithms enter the flux.

\section{B. Adiabatic Padé approximants}

\section{Center-of-mass energy}

Damour, Iyer, and Sathyaprakash [21] (henceforth DIS) proposed a new class of approximate waveforms constructed by introducing new energy and flux functions and by applying Padé summation [44] to build successive approximants to these two functions (henceforth $\mathrm{P}$ approximants). Their motivation for introducing these new func- tions and using their $\mathrm{P}$ approximants came from an examination of the behavior of the standard PN expansion and the new P approximants in the test-mass limit in which the exact gravitational energy flux is known numerically [45], the PN expansion of the flux is known through 5.5PN order [46], and the center-of-mass energy is known analytically as

$$
\frac{E\left(v_{\Omega} ; \nu=0\right)}{\mu}=\frac{1-2 v_{\Omega}^{2}}{\sqrt{1-3 v_{\Omega}^{2}}}-1,
$$

where $\mu=M \nu$ is the reduced mass.

DIS first observed that in the quantum two-body problem the symmetric quantity

$$
\epsilon \equiv \frac{E_{\mathrm{tot}}^{2}-m_{1}^{2}-m_{2}^{2}}{2 m_{1} m_{2}}
$$

(where the total relativistic energy $E_{\text {tot }}=E+M$ ), is the best energy function when treating the two-body problem as an effective one-body problem in an external field. Because in the test-mass limit

$$
\epsilon\left(v_{\Omega} ; \nu=0\right)=\frac{1-2 v_{\Omega}^{2}}{\sqrt{1-3 v_{\Omega}^{2}}},
$$

DIS defined the new energy function as

$$
e\left(v_{\Omega}\right) \equiv \epsilon^{2}-1
$$

as this function has a simple pole singularity on the real axis in the test-mass limit, and DIS conjectured that such a pole would continue to exist in the comparable-mass case. ${ }^{4}$ The energy function $E\left(v_{\Omega}\right)$ entering the balance equation (12) can be expressed in terms of $e\left(v_{\Omega}\right)$ as

$$
E\left(v_{\Omega}\right)=\left\{M^{2}+2 \nu M^{2}\left[\sqrt{1+e\left(v_{\Omega}\right)}-1\right]\right\}^{1 / 2}-M
$$

by combining Eqs. (29) and (31). [Note that the map between the adiabatic functions $e$ and $E$ given by Eq. (32) is the same map found in the EOB model between the effective Hamiltonian $H^{\text {eff }}$ and the real Hamiltonian $H^{\text {real }}$, as given by Eq. (44).]

Finally, DIS proposed as approximants to the energy function $e\left(v_{\Omega}\right)$ the diagonal or subdiagonal $\mathrm{P}$ approximants, depending on whether the PN order is even or odd. ${ }^{5}$ Investigating the behavior of the $\mathrm{P}$ approximants under variations of an (at the time) unknown coefficient in the 3PN center-of-mass energy, Damour, Jaranowski, and

\footnotetext{
${ }^{4}$ A motivation for using Eq. (31) instead of Eq. (29) as a basic quantity is that the former (unlike the latter) is amenable to Padé summation in the test-mass limit.

${ }^{5}$ As the energy is only a function of even powers of $v_{\Omega}$, the choice of using diagonal or subdiagonal (superdiagonal) is based on the order of $v_{\Omega}^{2}$ that is retained. For notational consistency, the indices on all approximants will refer to the power of $v_{\Omega}$. Other references define the indices on the energy approximants with respect to $v_{\Omega}^{2}$.
} 
Schäfer [24] found it more robust to use the superdiagonal $\mathrm{P}$ approximant instead of the subdiagonal $\mathrm{P}$ approximant at 3PN order. ${ }^{6}$ This suggestion was also adopted in Ref. [32] and will be used here; that is, we use subdiagonal $\mathrm{P}$ approximants for $1 \mathrm{PN}$, diagonal for $2 \mathrm{PN}$, and superdiagonal for 3PN.

The $\mathrm{P}$ approximants for the center-of-mass energy are defined as

$$
E_{p}^{q}\left(v_{\Omega}\right)=\left\{M^{2}+2 \nu M^{2}\left[\sqrt{1+e_{p}^{q}\left(v_{\Omega}\right)}-1\right]\right\}^{1 / 2}-M,
$$

where at $2 \mathrm{PN}$ order [21]

$$
e_{2}^{2}\left(v_{\Omega}\right)=-v_{\Omega}^{2} \frac{1+\frac{1}{3} \nu-\left(4-\frac{9}{4} \nu+\frac{1}{9} \nu^{2}\right) v_{\Omega}^{2}}{1+\frac{1}{3} \nu-\left(3-\frac{35}{12} \nu\right) v_{\Omega}^{2}},
$$

and at 3PN order [24]

$$
\begin{aligned}
e_{2}^{4}\left(v_{\Omega}\right)= & -v_{\Omega}^{2} \frac{1}{1-w_{3}(\nu) v_{\Omega}^{2}}\left[1-\left(1+\frac{1}{3} \nu+w_{3}(\nu)\right) v_{\Omega}^{2}\right. \\
& \left.-\left(3-\frac{35}{12} \nu-\left(1+\frac{1}{3} \nu\right) w_{3}(\nu)\right) v_{\Omega}^{4}\right]
\end{aligned}
$$

where

$$
\begin{aligned}
w_{3}(\nu)= & \frac{40}{36-35 \nu}\left[\frac{27}{10}+\frac{1}{16}\left(\frac{41}{4} \pi^{2}-\frac{4309}{15}\right) \nu\right. \\
& \left.+\frac{103}{120} \nu^{2}-\frac{1}{270} \nu^{3}\right] .
\end{aligned}
$$

\section{Gravitational-wave energy flux}

As originally pointed out in Refs. [49,50], the flux function in the test-mass limit has a simple pole at the light-ring position (i.e., the last unstable circular orbit of a photon). Motivated by this, DIS introduced a new flux-type function

$$
f_{k}\left(v_{\Omega}\right)=\left(1-\frac{v_{\Omega}}{v_{\text {pole }}(\nu)}\right) F_{k}\left(v_{\Omega} ; \nu\right)
$$

with the suggestion that $v_{\text {pole }}$ be chosen to be at the light ring (pole singularity) of the new energy function.

In order to construct well-behaved approximants, DIS proposed to normalize the velocity $v_{\Omega}$ entering the logarithms in Eq. (26) to some relevant scale which they chose to be $v_{\text {lso }}(\nu)$, where the last stable orbit (LSO) is defined as the minimum of the energy. Also, they factored out the logarithms yielding

${ }^{6}$ Subdiagonal $\mathrm{P}$ approximants were extended to 3PN order in Ref. [47], and LAL [48] software uses those P approximants for the energy function.

$$
\begin{aligned}
f_{k}\left(v_{\Omega}\right)= & \frac{32}{5} \nu^{2} v_{\Omega}^{10}\left[1+\log \frac{v_{\Omega}}{v_{\mathrm{lso}}(\nu)}\left(\sum_{i \geq 6}^{k} \ell_{i} v_{\Omega}^{i}\right)\right] \\
& \times\left(1-\frac{v_{\Omega}}{v_{\text {pole }}(\nu)}\right) \sum_{i=0}^{k} \mathcal{F}_{i}^{\log -\mathrm{fac}} v_{\Omega}^{i},
\end{aligned}
$$

where $\ell_{i}$ and $\mathcal{F}_{i}^{\log \text {-fac }}$ are functions of $\mathcal{F}_{i}$. Through 3.5PN order, $\ell_{6}=-1712 / 105, \ell_{7}=0$, and $\mathcal{F}_{i}^{\text {log-fac }}=\mathcal{F}_{i}$ with the replacement of $v_{\Omega} \rightarrow v_{\text {lso }}$ in $\mathcal{F}_{6}$ [see Eq. (26)].

Finally, DIS proposed to define the $\mathrm{P}$ approximant of the GW energy flux as

$$
F_{n}^{m}\left(v_{\Omega}\right)=\frac{1}{1-v_{\Omega} / v_{\text {pole }}(\nu)} f_{n}^{m}\left(v_{\Omega}\right),
$$

where

$$
\begin{aligned}
f_{n}^{m}\left(v_{\Omega}\right)= & \frac{32}{5} \nu^{2} v_{\Omega}^{10}\left[1+\log \frac{v_{\Omega}}{v_{\mathrm{lso}}(\nu)}\left(\sum_{i \geq 6}^{k} \ell_{i} v_{\Omega}^{i}\right)\right] \\
& \times \mathrm{P}_{n}^{m}\left[\left(1-\frac{v_{\Omega}}{v_{\text {pole }}(\nu)}\right) \sum_{i=0}^{k} \mathcal{F}_{i}^{\log -\mathrm{fac}} v_{\Omega}^{i}\right],
\end{aligned}
$$

and $\mathrm{P}_{n}^{m}[x]$ denotes Padé summation of the series $x$. DIS proposed to use the diagonal or subdiagonal $\mathrm{P}$ approximants, depending on whether $k=n+m$ is even or odd. Furthermore, DIS proposed to use $v_{\text {lso }}(\nu)$ and $v_{\text {pole }}(\nu)$ as the minimum and pole of the center-of-mass energy $\mathrm{P}$ approximant of the same PN order. At 2PN (the order to which the PN expansion was known by DIS) $v_{\text {pole }}$ is determined from the pole of the Padé energy function $e_{2}^{2}$, yielding

$$
v_{\mathrm{pole}}^{2 \mathrm{PN}}(\nu)=\frac{1}{\sqrt{3}} \sqrt{\frac{1+\frac{1}{3} \nu}{1-\frac{35}{36} \nu}} .
$$

When the PN expansion was extended to 3PN order, it was found that none of the 3PN P approximants have a physical pole. Therefore, somewhat arbitrarily, we will follow previous analyses and use the value (41) also at $3 \mathrm{PN}$ order. We denote the $\mathrm{P}$ approximants defined by Eqs. (33) and (39) as $F_{n}^{m} / E_{p}^{q}$.

The denominator in the Padé summation of the GW energy flux can have zeros. They are called extraneous poles of the $\mathrm{P}$ approximant [44]. It is desirable that these poles be located at high frequency (i.e., beyond the transition from inspiral to plunge). We shall see that depending on the PN order and also the mass ratio, extraneous poles can be present at low frequencies. This could indicate poor convergence of the Padé summation.

In Secs. IV B, VI B, and VIC we shall investigate how to improve the closeness of the PN approximants to the numerical data by varying $a_{5}[16,18,26], v_{\text {pole }}[18,26]$, and also by introducing higher-order PN coefficients in the flux function. When varying $v_{\text {pole }}$ in the $\mathrm{P}$ approximant at 3.5PN order, extraneous poles appear at low values of 
$v_{\Omega}$. Therefore, in order to push these poles to very high frequency, we follow the suggestion of Ref. [18], and use P approximants at $4 \mathrm{PN}$ order, where the $4 \mathrm{PN}$ coefficient is set to its known value in the test-mass limit. This cure may fail for different mass ratios if new extraneous poles appear at low frequency. Furthermore, the logarithm in the flux is not factored out as in Eq. (38), but treated as a constant when Padé summation is done. In this case, the flux function is denoted $\bar{F}_{n}^{m}$.

We notice that DIS motivated the introduction of the $\mathrm{P}$ approximants first in the test-mass limit case by observing much faster and monotonic convergence of the Padé energy, flux, and waveforms with respect to Taylor energy, flux, and waveforms. Quantitative tests of the convergence were done only for the Padé waveforms (see e.g., Tables III and IV in Ref. [21]), while for the flux and the energy conclusions were drawn qualitatively from Figs. 3 and 4 of Ref. [21]. DIS then conjectured that the comparable-mass case is a smooth deformation of the test-mass limit case, and proposed to use close-to-diagonal $\mathrm{P}$ approximants for the flux and the energy when $\nu \neq 0$. In the Appendix, we perform a few convergence tests of the $\mathrm{P}$ approximants of the flux function in the test-mass limit case, and conclude that whereas the $\mathrm{P}$ approximants provide a better fit to the numerical flux at 5.5PN order, they do not accelerate the convergence of the Taylor series expansion of the energy flux.

\section{Nonadiabatic effective-one-body approximants}

The EOB model goes beyond the adiabatic approximation and can incorporate deviations from the Keplerian law when the radial separation become smaller than the last stable circular orbit.

Here we briefly review the main equations defining the EOB dynamics and refer the reader to previous papers for more details $[15,16,18,19,22-24,33]$. The nonspinning EOB effective Hamiltonian is [22,24]:

$$
\begin{aligned}
H^{\mathrm{eff}}(\mathbf{r}, \boldsymbol{p})= & \mu \hat{H}^{\mathrm{eff}}(\mathbf{r}, \mathbf{p}) \\
= & \mu\left\{A ( r ) \left[1+\mathbf{p}^{2}+\left(\frac{A(r)}{D(r)}-1\right)(\mathbf{n} \cdot \mathbf{p})^{2}\right.\right. \\
& \left.\left.+\frac{1}{r^{2}} 2(4-3 \nu) \nu(\mathbf{n} \cdot \mathbf{p})^{4}\right]\right\}^{1 / 2},
\end{aligned}
$$

with $\mathbf{r}$ and $\mathbf{p}$ being the reduced dimensionless variables; $\mathbf{n}=\mathbf{r} / r$ where we set $r=|\mathbf{r}|$. In absence of spins, the motion is constrained to a plane. Introducing polar coordinates $\left(r, \Phi, p_{r}, p_{\Phi}\right)$, the EOB effective metric reads

$$
\begin{aligned}
d s_{\mathrm{eff}}^{2} & \equiv g_{\mu \nu}^{\mathrm{eff}} d x^{\mu} d x^{\nu} \\
& =-A(r) c^{2} d t^{2}+\frac{D(r)}{A(r)} d r^{2}+r^{2}\left(d \theta^{2}+\sin ^{2} \theta d \phi^{2}\right) .
\end{aligned}
$$

The EOB real Hamiltonian is

$$
H^{\text {real }}=M \sqrt{1+2 \nu\left(\frac{H^{\mathrm{eff}}-\mu}{\mu}\right)}-M
$$

and we define $\hat{H}^{\text {real }}=H^{\text {real }} / \mu$. The T approximants to the coefficients $A(r)$ and $D(r)$ in Eqs. (42) and (43) read $[22,24]$

$$
\begin{aligned}
& A_{k}(r)=\sum_{i=0}^{k+1} \frac{a_{i}}{r^{i}}, \\
& D_{k}(r)=\sum_{i=0}^{k} \frac{d_{i}}{r^{i}},
\end{aligned}
$$

where

$$
\begin{gathered}
a_{0}=1, \quad a_{1}=-2, \quad a_{2}=0, \\
a_{3}(\nu)=2 \nu, \quad a_{4}(\nu)=\left(\frac{94}{3}-\frac{41}{32} \pi^{2}\right) \nu, \\
d_{0}=1, \quad d_{1}=0, \quad d_{2}(\nu)=-6 \nu, \\
d_{3}(\nu)=2(3 \nu-26) \nu .
\end{gathered}
$$

In Sec. VIC, we will explore the flexibility of the EOB model by tuning the pseudo 4PN order coefficients $a_{5}(\nu)$ which we will take to have the following functional form ${ }^{7}$

$$
a_{5}(\nu)=a_{5} \nu
$$

In order to assure the presence of an horizon in the effective metric, we need to factor out a zero of $A(r)$. This is obtained by applying the Padé summation [24]. Thus, the coefficients $A_{k}(r)$ and $D_{k}(r)$ are replaced by the Padé approximants [24]

$$
A_{2}^{1}(r)=\frac{r(-4+2 r+\nu)}{2 r^{2}+2 \nu+r \nu}
$$

at $2 \mathrm{PN}$ order, and

$$
A_{3}^{1}(r)=\frac{\operatorname{Num}\left(A_{3}^{1}\right)}{\operatorname{Den}\left(A_{3}^{1}\right)}
$$

with

$$
\operatorname{Num}\left(A_{3}^{1}\right)=r^{2}\left[\left(a_{4}(\nu)+8 \nu-16\right)+r(8-2 \nu)\right],
$$

and

$$
\begin{aligned}
\operatorname{Den}\left(A_{3}^{1}\right)= & r^{3}(8-2 \nu)+r^{2}\left[a_{4}(\nu)+4 \nu\right] \\
& +r\left[2 a_{4}(\nu)+8 \nu\right]+4\left[\nu^{2}+a_{4}(\nu)\right],
\end{aligned}
$$

at $3 \mathrm{PN}$ order. When exploring the flexibility of the EOB model, we use the following Padé approximant at 4 PN order $[16,26]$ :

\footnotetext{
${ }^{7}$ Note that what we denote $a_{5}$ in this paper was denoted $\lambda$ in Ref. [16].
} 


$$
A_{4}^{1}(r)=\frac{\operatorname{Num}\left(A_{4}^{1}\right)}{\operatorname{Den}\left(A_{4}^{1}\right)},
$$

with

$$
\begin{aligned}
\operatorname{Num}\left(A_{4}^{1}\right)= & r^{3}\left[32-24 \nu-4 a_{4}(\nu)-a_{5}(\nu)\right] \\
& +r^{4}\left[a_{4}(\nu)-16+8 \nu\right],
\end{aligned}
$$

and

$$
\begin{aligned}
\operatorname{Den}\left(A_{4}^{1}\right)= & -a_{4}^{2}(\nu)-8 a_{5}(\nu)-8 a_{4}(\nu) \nu+2 a_{5}(\nu) \nu \\
& -16 \nu^{2}+r\left[-8 a_{4}(\nu)-4 a_{5}(\nu)\right. \\
& \left.-2 a_{4}(\nu) \nu-16 \nu^{2}\right]+r^{2}\left[-4 a_{4}(\nu)-2 a_{5}(\nu)\right. \\
& -16 \nu]+r^{3}\left[-2 a_{4}(\nu)-a_{5}(\nu)-8 \nu\right] \\
& +r^{4}\left[-16+a_{4}(\nu)+8 \nu\right] .
\end{aligned}
$$

For the coefficient $D(r)$, the $\mathrm{P}$ approximant used at $2 \mathrm{PN}$, $3 \mathrm{PN}$, and 4PN order, respectively, are $[16,24,26]$ :

$$
\begin{gathered}
D_{2}^{0}(r)=1-\frac{6 \nu}{r^{2}}, \\
D_{3}^{0}(r)=\frac{r^{3}}{r^{3}+6 \nu r+2 \nu(26-3 \nu)}, \\
D_{4}^{0}(r)=\frac{r^{4}}{r^{4}+6 \nu r^{2}+2 \nu(26-3 \nu) r-d_{4}(\nu)+36 \nu^{2}},
\end{gathered}
$$

and we choose somewhat arbitrarily $d_{4}(\nu)=36 \nu^{2}$, so that $D_{4}^{0}=D_{3}^{0}$. (We note that the value of $d_{4}$ does not affect much the EOB evolution [16].) The EOB Hamilton equations written in terms of the reduced quantities $\hat{H}^{\text {real }}$ and $\hat{t}=t / M, \hat{\Omega}=\Omega M[23]$, are

$$
\begin{gathered}
\frac{d r}{d \hat{t}}=\frac{\partial \hat{H}^{\text {real }}}{\partial p_{r}}\left(r, p_{r}, p_{\Phi}\right), \\
\frac{d \Phi}{d \hat{t}} \equiv \hat{\Omega}=\frac{\partial \hat{H}^{\text {real }}}{\partial p_{\Phi}}\left(r, p_{r}, p_{\Phi}\right), \\
\frac{d p_{r}}{d \hat{t}}=-\frac{\partial \hat{H}^{\text {real }}}{\partial r}\left(r, p_{r}, p_{\Phi}\right), \\
\frac{d p_{\Phi}}{d \hat{t}}=\hat{\mathcal{F}}\left[\hat{\Omega}\left(r, p_{r}, p_{\Phi}\right)\right],
\end{gathered}
$$

where for the $\Phi$ component of the radiation-reaction force a few approximants are available. Originally, Ref. [23] suggested the following Keplerian P approximants to the flux

$$
\mathrm{K} \hat{\mathcal{F}}_{n}^{m} \equiv-\frac{1}{\nu v_{\Omega}^{3}} F_{n}^{m}\left(v_{\Omega} ; \nu, v_{\text {pole }}\right),
$$

where $F_{n}^{m}$ is given by the Padé flux in Eqs. (39) and (40).
Here, by Keplerian, we mean that in the flux the tangential velocity $V_{\Phi}=\dot{\Phi} r$ is set to $V_{\Phi} \equiv v_{\Omega}=\dot{\Phi}^{1 / 3}$, having assumed the Keplerian relation $\dot{\Phi}^{2} r^{3}=1$. It was then pointed out in Ref. [34] that the Keplerian relation becomes less and less accurate once the binary passes through the last stable orbit. A more appropriate approximant to the flux would be

$$
\mathrm{nK} \hat{\mathcal{F}}_{n}^{m} \equiv-\frac{v_{\Omega}^{3}}{\nu V_{\Phi}^{6}} F_{n}^{m}\left(V_{\Phi} ; \nu, v_{\text {pole }}\right),
$$

where $V_{\Phi} \equiv \dot{\Phi} r_{\Omega}$. Notice that because the EOB Hamiltonian is a deformation of the Schwarzschild Hamiltonian, the exact Keplerian relation is $\dot{\Phi}^{2} r_{\Omega}^{3}=1$ with $r_{\Omega} \equiv r\left[\psi\left(r, p_{\Phi}\right)\right]^{1 / 3}$, and $\psi$ is defined following the argument presented around Eqs. (19) to (22) in Ref. [34]:

$$
\begin{aligned}
\frac{1}{\psi r^{3}} & \equiv \omega_{\text {circ }}^{2}=\left(\frac{\partial \mathcal{H}\left(r, p_{r}=0, p_{\phi}\right)}{\partial p_{\phi}}\right)^{2} \\
& =\frac{1}{r^{3}} \frac{p_{\phi}^{2} A(r)}{\left(1+\frac{p_{\phi}^{2}}{r^{2}}\right) r\left(1+2 \eta\left(\sqrt{w\left(r, p_{\phi}\right)}-1\right)\right.},
\end{aligned}
$$

where $w\left(r, p_{\phi}\right)=A(r)\left(1+\frac{p_{\phi}^{2}}{r^{2}}\right)$. The value of $p_{\phi}$ of circular orbits are obtained by minimizing with respect to $r$ the circular orbit Hamiltonian $\mathcal{H}\left(r, p_{r}=0, p_{\phi}\right)$ and it yields the following relation between $r$ and $p_{\phi}$

$$
\frac{2 p_{\phi}^{2} A(r)}{r^{3}}=\left(1+\frac{p_{\phi}^{2}}{r^{2}}\right) \frac{d A(r)}{d r} .
$$

By inserting Eq. (67) in the definition of $\psi$, and replacing all $p_{\phi}$ except the one which implicitly appears in $w\left(r, p_{\phi}\right)$ we obtain

$$
\psi=\frac{1+2 \eta\left(\sqrt{w\left(r, p_{\phi}\right)}-1\right)}{\frac{1}{2} r^{2} d A(r) / d r} .
$$

Finally, Refs. [32,33] introduced another possible variation of the EOB flux approximants which use T approximants for the flux given by Eq. (19), in either the Keplerian or non-Keplerian form, i.e.

$$
\mathrm{K} \hat{\mathcal{F}}_{n}=-\frac{1}{\nu v_{\Omega}^{3}} F_{n}\left(v_{\Omega}\right),
$$

and

$$
\mathrm{nK} \hat{\mathcal{F}}_{n}=-\frac{v_{\Omega}^{3}}{\nu V_{\Phi}^{6}} F_{n}\left(V_{\Phi}\right) .
$$

Note that the flux for the non-Keplerian EOB models are not simply functions of the orbital frequency $\Omega$. We denote the original E approximants [22-24] which use the Padé flux (40) as $F_{n}^{m} / H_{p}$ where $H_{p}$ is $H^{\text {real }}$ computed from $A_{p}^{1}$ and $D_{p}^{0}$. Other E approximants used in this paper are summarized in Table I. The initial conditions for 
Eqs. (60)-(63) are obtained following Ref. [23] and starting the evolution far apart to reduce the eccentricity to negligible values.

\section{Waveforms}

The PN waveforms are obtained by substituting the orbital phase and frequency into the spherical harmonic mode $(2,2)$ with amplitude corrections through 3PN order [51-53]

$$
\begin{aligned}
h_{22}= & -8 \sqrt{\frac{\pi}{5}} \frac{\nu M}{R} e^{-2 i \Phi} v_{\Omega}^{2}\left\{1-v_{\Omega}^{2}\left(\frac{107}{42}-\frac{55}{42} \nu\right)\right. \\
& +2 \pi v_{\Omega}^{3}-v_{\Omega}^{4}\left(\frac{2173}{1512}+\frac{1069}{216} \nu-\frac{2047}{1512} \nu^{2}\right) \\
& -v_{\Omega}^{5}\left[\left(\frac{107}{21}-\frac{34}{21} \nu\right) \pi+24 i \nu\right]+v_{\Omega}^{6}\left[\frac{27027409}{646800}\right. \\
& -\frac{856}{105} \gamma_{E}+\frac{2}{3} \pi^{2}-\frac{1712}{105} \ln 2-\frac{856}{105} \ln v_{\Omega} \\
& -\left(\frac{278185}{33264}-\frac{41}{96} \pi^{2}\right) \nu-\frac{20261}{2772} \nu^{2}+\frac{114635}{99792} \nu^{3} \\
& \left.\left.+\frac{428 i}{105} \pi\right]+O\left(\epsilon^{7 / 2}\right)\right\} .
\end{aligned}
$$

For the adiabatic models, the orbital phase is obtained by rewriting the energy-balance equation (12) as

$$
\frac{d \Omega}{d t}=-\frac{F}{d E / d \Omega},
$$

and integrating this equation along with $d \Phi / d t=\Omega$. The Taylor approximants are formed first by substituting $F=$ $F_{n}$ and $E=E_{n}$ into Eq. (72). The P-approximant waveform is formed similarly by substituting $F=F_{n}^{m}$ and $E=$ $E_{n}^{m}$ into Eq. (72). The TaylorT1 and Padé approximants then numerically integrate Eq. (72). The TaylorT4 approximant is formed by first reexpanding the right side of Eq. (72) as a single Taylor expansion truncated at the appropriate order, and then numerically integrating the resulting equation. The TaylorT2 and TaylorT3 approximants perform the integration analytically. The various Taylor approximants are reviewed in Sec. III E of Ref. [12].

For the nonadiabatic EOB models, the orbital phase is determined by solving Hamilton's equations (60)-(63).

After computing $h_{22}$, the appropriate time derivatives are taken to form $\dot{h}_{22}$ and $\Psi_{4}^{22}$.

\section{COMPARISON WITH POST-NEWTONIAN APPROXIMANTS: ENERGY FLUX}

We now compare the numerical GW energy flux with predictions from PN theory. In Sec. IVA, we present comparisons with $\mathrm{T}, \mathrm{P}$, and $\mathrm{E}$ approximants, and, in Sec. IV B, we explore ways of fitting the numerical flux by introducing higher-order PN coefficients and varying the value of $v_{\text {pole }}$ away from $v_{\text {pole }}^{2 \mathrm{PN}}$ [Eq. (41)].
The PN flux is derived as a function of frequency, so it is natural to perform this comparison as a function of frequency. One alternative, comparison as a function of time, would require computation of the $\mathrm{PN}$ phase as a function of time. This depends on the PN energy, so that a comparison with respect to time would mix effects due to flux and energy. Furthermore, comparisons with respect to time are sensitive to (and likely dominated by) secularly accumulating phase differences [54].

The PN flux is given in terms of the orbital frequency $\Omega$-see Eqs. (13) and (19)—so at first glance, it might seem natural to compare PN and NR energy fluxes at particular values of $\Omega$. However, the orbital frequency is gauge dependent, and there is no simple relation between the NR orbital frequency and the PN orbital frequency. Nor is there a simple relation between the NR orbital frequency and any quantity measured at infinity (where the energy flux is defined). In particular, it is very difficult to determine the NR orbital frequency as a function of retarded time. In contrast, the frequency $\varpi$ [see Eq. (5)] of the GWs at infinity is an observable quantity, and is easily obtained from both PN formulae and from the NR simulation. Therefore, to achieve a meaningful comparison, we compare the PN and NR energy flux at particular values of $\varpi$.

In order to compute the PN flux as a function of $\varpi$, we need to find the mapping $\varpi_{\mathrm{PN}}: \Omega \rightarrow \varpi$. In order to find this mapping, we must build a PN waveform as a function of $\Omega$ and compute $\varpi$ as defined by Eq. (5). We construct the waveforms as described in Sec. III D. For the T approximant of the flux, we will use the TaylorT4 waveform. In Fig. 5, we plot both GW frequencies [defined in Eqs. (4)

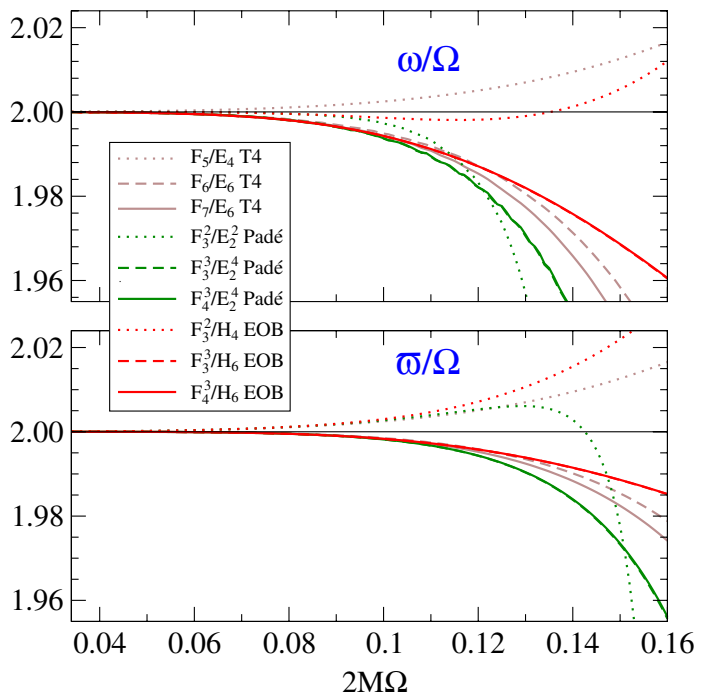

FIG. 5 (color online). Ratio of GW frequencies $\omega$ and $\varpi$ to orbital frequency, as a function of (twice) the orbital frequency, for different PN models. The GW frequencies $\omega$ and $\varpi$ are defined in Eqs. (4) and (5). Solid lines correspond to 3.5PN; dashed and dotted lines correspond to $3 \mathrm{PN}$ and $2.5 \mathrm{PN}$, respectively. 
and (5)]. We then invert the mapping to obtain $\Omega_{\mathrm{PN}}=$ $\varpi_{\mathrm{PN}}^{-1}: \varpi \rightarrow \Omega$. So, given the PN flux $F(\Omega)$ from Sec. III, the flux as a function of the GW frequency is given by $F(\varpi)=$ $F\left(\Omega_{\mathrm{PN}}(\varpi)\right)$. The relation $\Omega_{\mathrm{PN}}(\varpi)$ depends on the instantaneous evolution of the PN model around frequency $\Omega$, and is therefore (unfortunately) dependent on the PN model, in particular, the choice of PN energy. This dependence, however, is local and will not lead to secularly accumulating differences.

Notice from Fig. 5 that the orbital frequency and the GW frequency differ by $\sim 1 \%-3 \%$ at large frequencies, depending on the PN model and the PN order, and the difference in $\varpi$ between different PN models is about $5 \%$. Because the energy flux is roughly proportional to $\varpi^{10 / 3}$ [more precisely, $d \log F / d \log (M \varpi)$ increases to $\sim 3.6$ at $M \varpi=$ $0.15]$, the difference in the flux caused by using GW frequency from different PN models is about 3 to 4 times the difference in GW frequencies. Figure 6 illustrates this effect by intentionally plotting the PN flux versus the incorrect frequency $\Omega$. Because changing the PN model has a significant effect on the flux, we consider flux comparisons for several different PN models below.

Note that for the flux comparison (and the comparisons of the derivative of the energy in Sec. V), the PN waveforms are used only to define the mapping between $\Omega$ and $\varpi$. The PN flux is taken directly from the PN flux expressions, e.g., Eq. (19), and not computed by applying Eq. (3) to PN waveforms $h(t)$. Equation (3) is used only to compute the numerical flux.

\section{A. Flux comparison}

Figure 7 plots the NR flux and the fluxes for the T, P, and $\mathrm{E}$ approximants at 3.5PN order as a function of the GW frequency $\varpi$ computed from $\dot{h}_{22}$. The T approximant is TaylorT4 [12]. Along the top of this figure (as in several figures below) we indicate the number of gravitationalwave cycles up to merger, where we define "merger" as

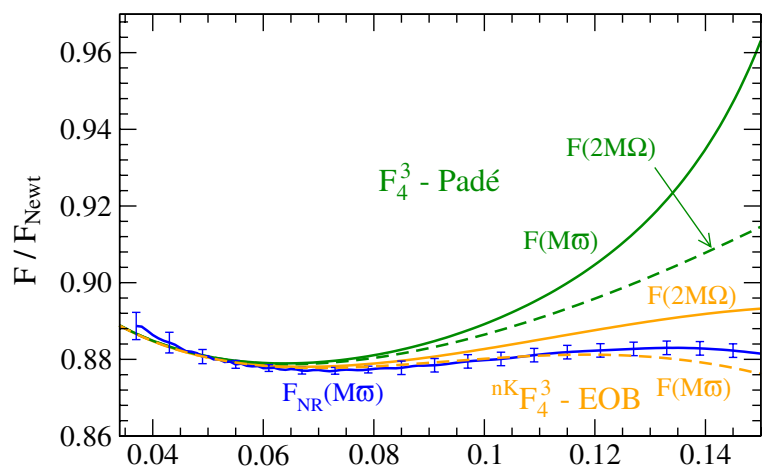

FIG. 6 (color online). Effect of choice of frequency. Shown are the PN fluxes for two representative PN approximants, plotted (correctly) as function of $\varpi$ and (incorrectly) as function of $2 \Omega$. Plotting as a function of $2 \Omega$ changes the PN fluxes significantly relative to the numerical flux $F_{\mathrm{NR}}$.
GW cycles before merger

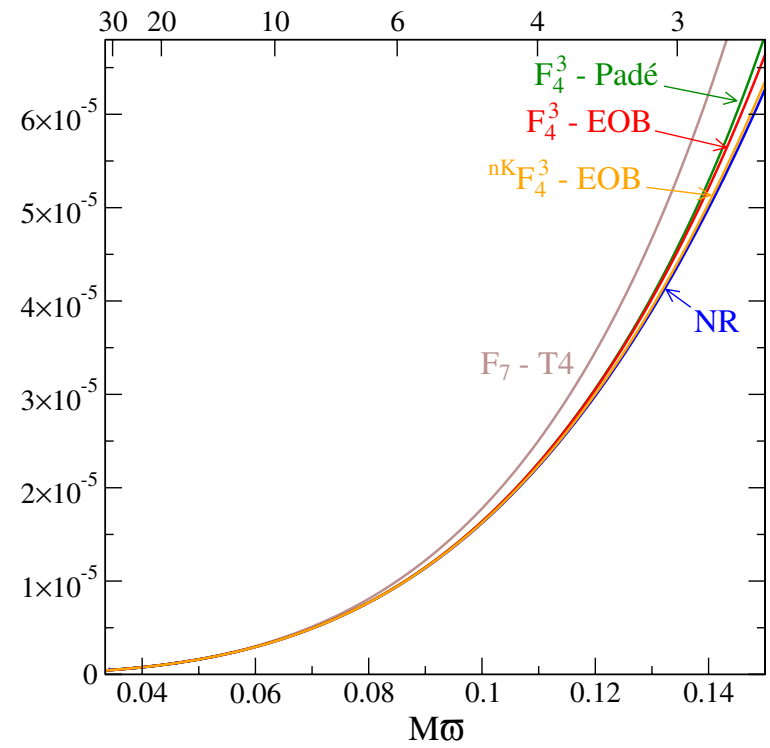

FIG. 7 (color online). Comparison between the numerical energy flux and several PN approximants at 3.5PN order versus GW frequency $\varpi$ extracted from $\dot{h}_{22}$ in the equal-mass case.

the maximum of $\left|\Psi_{4}^{22}\right|$. Figure 8 zooms over the first 15 $\mathrm{GW}$ cycles. We notice that during the first $15 \mathrm{GW}$ cycles

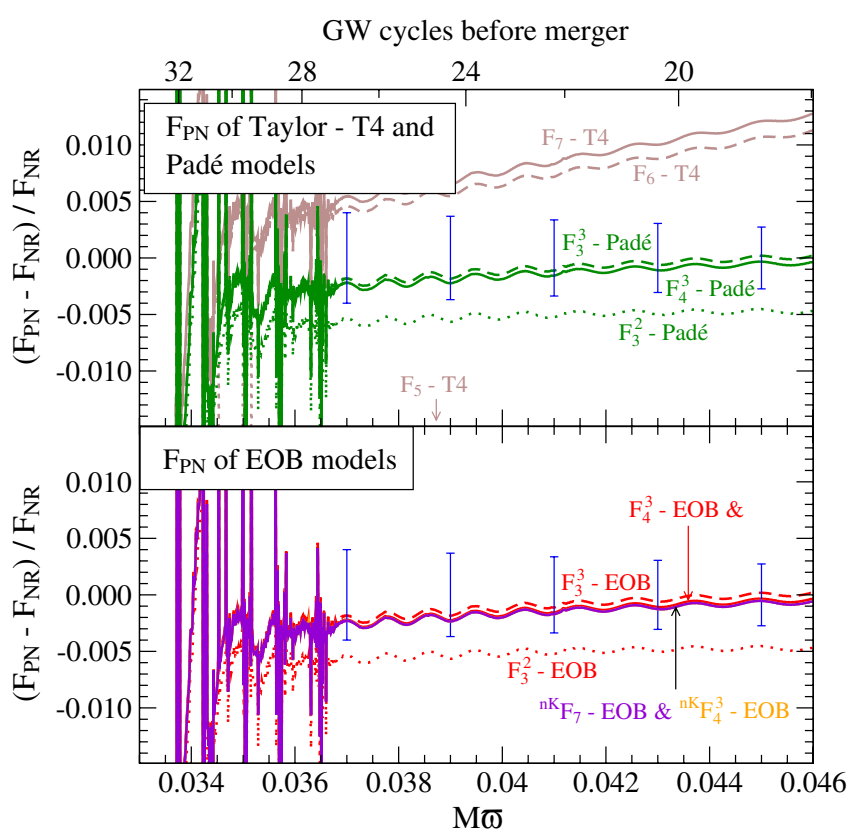

FIG. 8 (color online). Comparison between the numerical energy flux and several PN approximants versus GW frequency $\varpi$ extracted from $\dot{h}_{22}$ in the equal-mass case. We show the relative difference between numerical flux and PN flux, as well as the estimated error of the numerical flux (blue bars; see Fig. 2). Solid lines represent 3.5PN models and NR; dashed and dotted lines correspond to 3PN and 2.5PN models, respectively. For notation see Table I and caption therein. 


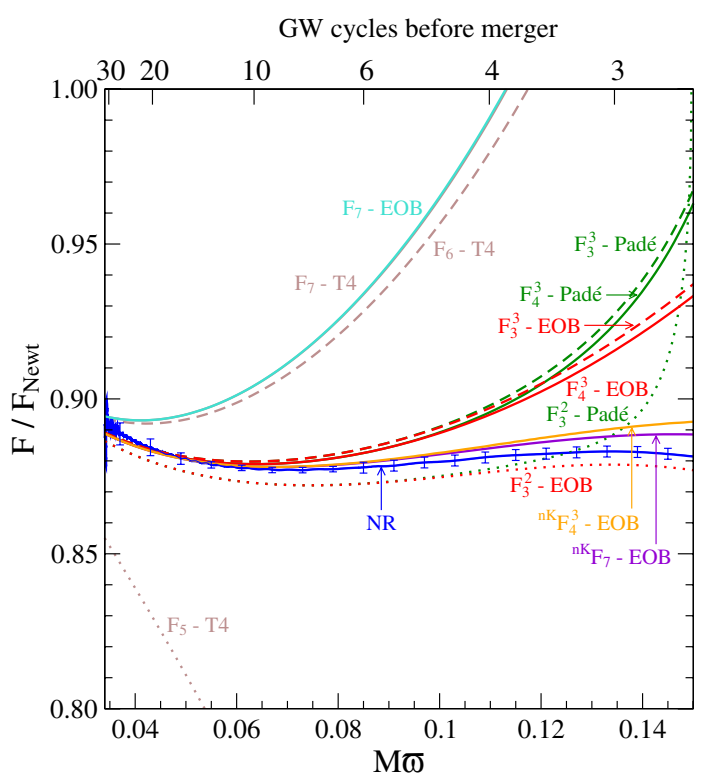

FIG. 9 (color online). Comparison of normalized energy flux $F / F_{\text {Newt }}$ [see Eq. (73)] for the equal-mass case. Solid lines represent 3.5PN models and NR; dashed and dotted lines correspond to 3PN and 2.5PN models, respectively. For notation see Table I and caption therein.

the numerical data are fit best by the $\mathrm{P}$ and $\mathrm{E}$ approximants at $3 \mathrm{PN}$ and 3.5PN order. At these low frequencies the NR flux is best matched by the Keplerian and non-Keplerian EOB models and the Padé model.

To more clearly show the behavior of the PN approximants, we plot in Fig. 9 the energy flux normalized by the Newtonian flux. The normalized flux is computed as

$$
\frac{F(\varpi)}{F_{\text {Newt }}(\varpi)} \equiv \frac{F(\varpi)}{\frac{32}{5} \nu^{2}\left(\frac{M \varpi}{2}\right)^{10 / 3}},
$$

where for the same reason mentioned above, the Newtonian flux is expressed in terms of the GW frequency. Notice that the $\mathrm{P}$ approximants and some of the E approximants use the same Padé flux, but they start differing at $M \varpi \sim 0.12$ due to their different GW frequencies (obtained from an adiabatic and nonadiabatic evolution, respectively). The E approximants with Keplerian and nonKeplerian flux increase less abruptly at high frequency than the $\mathrm{P}$ and $\mathrm{T}$ approximants. This is a consequence of nonadiabatic effects captured by the EOB model. Quite remarkably, the E approximants with non-Keplerian fluxes are rather close to the NR result for the entire range of frequency spanned by the simulation. ${ }^{8}$ We observe that

\footnotetext{
${ }^{8}$ We notice that whereas the Keplerian Padé-based (or Taylorbased) approximants to the flux differ from each other only when expressed in terms of the GW frequency, the non-Keplerian Padé-based (or Taylor-based) approximants to the flux differ from the others because their functional dependence on the frequency is different [e.g., compare Eq. (65) with Eq. (64)].
}

somewhat accidentally the PN approximants at $2.5 \mathrm{PN}$ order are also close to the numerical flux.

The normalized NR flux starts to decrease at $M \varpi \sim$ 0.13 . We notice that this behavior is rather different from the behavior of the normalized flux in the test-mass limit (see Fig. 19 in the Appendix). The E approximants with non-Keplerian Padé or Taylor flux show a similar decreasing behavior at high frequency.

Both Figs. 8 and 9 show that in the equal-mass case $\mathrm{P}$ approximants fit the numerical results better than $\mathrm{T}$ approximants. In numerical analysis, however, Padé summation is often used as a technique to accelerate the convergence of a slowly converging Taylor series (e.g., see Tables 8.9 and 8.12 in Ref. [44]); hence it is natural to ask in the PN case whether Padé summation indeed accelerates the convergence of the series. In Table II, we list the $\mathrm{T}$ and $\mathrm{P}$ approximants of $F / F_{\text {Newt }}$ computed at subsequent PN orders and for several values of $v_{\Omega}$ [from left to right $v_{\Omega}=0.1,0.25$ (i.e., beginning of the numerical simulation), 0.3, 0.35, and 0.4.] In Fig. 10, we perform a Cauchy convergence test and compute the difference between $\mathrm{T}$ and $\mathrm{P}$ approximants at subsequent PN orders. The figures do not suggest an acceleration of the convergence. We notice that in the equal-mass case $\mathrm{P}$ approximants are converging more systematically than $\mathrm{T}$ approximants. However, this fact seems to depend on the mass ratio, as can be seen by comparing Fig. 10 with Table IV and Fig. 21 in the Appendix which are obtained in the test-mass limit.

\section{B. On the fitting of the numerical relativity energy flux}

In view of building accurate analytical templates that can interpolate the NR waveforms during inspiral, merger, and ringdown, we explore here the possibility of improving the PN approximants to the energy flux by introducing phenomenological higher-order PN coefficients and/or by varying the value of $v_{\text {pole }}$. This study should be considered a first exploration of the problem, demonstrating only the flexibility of the PN models. None of the quantities derived here should be used as the basis for further work.

We will minimize the difference between the PN flux and the numerical flux by varying particular coefficients in the PN model. Ideally, the PN and numerical fluxes should be expressed as functions of $\varpi$ before taking this difference, so that the fluxes are compared in a physically meaningful way. Unfortunately, the calculation of $\varpi$ for the PN models is time consuming, because for each trial value of the phenomenological coefficient it is necessary to compute a full waveform to determine the mapping between $\varpi$ and $\Omega$. So instead, in this section we simply compare PN and numerical fluxes as functions of $\Omega$, where we define the numerical orbital frequency as $\Omega \equiv \varpi / 2$. In Fig. 6, we can see that the error introduced by the discrepancy between $\Omega$ and $\varpi / 2$ will be significant. As we will show in Sec. VIB, the waveforms produced using these "tuned" flux functions will improve agreement with the numerical 
TABLE II. Normalized energy flux $F / F_{\text {Newt }}$ for the $\mathrm{T}$ and $\mathrm{P}$ approximants at subsequent $\mathrm{PN}$ orders for select velocities $v_{\Omega}$. $v_{\Omega}=$ 0.25 corresponds to the start of the numerical simulation. The P-approximant flux is given by Eq. (39). Note that the P approximant has an extraneous pole at $1 \mathrm{PN}$ order at $v_{\Omega}=0.326$. We use $v_{\text {lso }}=v_{\text {lso }}^{2 \mathrm{PN}}=0.4456$ and $v_{\text {pole }}=v_{\text {pole }}^{2 \mathrm{PN}}=0.6907$. We use boldface to indicate the range of significant figures that do not change with increasing PN order.

\begin{tabular}{|c|c|c|c|c|c|c|c|c|c|c|}
\hline $\begin{array}{l}\text { PN order } \\
(n+m) / 2\end{array}$ & $\begin{array}{c}v_{\Omega}=0.1,2 \\
\frac{F_{n+m}}{F_{\text {Newt }}}\end{array}$ & $\begin{array}{c}M \Omega=0.002 \\
\frac{F_{n}^{m}}{F_{\text {Newt }}} \\
\end{array}$ & $\begin{array}{c}v_{\Omega}=0.25, \\
\frac{F_{n+m}}{F_{\text {Newt }}} \\
\end{array}$ & $\begin{array}{c}M \Omega=0.031 \\
\frac{F_{n}^{m}}{F_{\text {Newt }}}\end{array}$ & $\begin{array}{c}v_{\Omega}=0.3, \\
\frac{F_{n+m}}{F_{\text {Newt }}} \\
\end{array}$ & $\begin{array}{c}M \Omega=0.054 \\
\frac{F_{n}^{m}}{F_{\text {Newt }}} \\
\end{array}$ & $\begin{array}{c}v_{\Omega}=0.35 \\
\frac{F_{n+m}}{F_{\text {Newt }}} \\
\end{array}$ & $\begin{array}{c}5,2 M \Omega=0.086 \\
\frac{F_{n}^{m}}{F_{\text {Newt }}} \\
\end{array}$ & $v_{\Omega}=0.4$, & $\begin{array}{c}2 M \Omega=0.128 \\
\frac{F_{n}^{m}}{F_{\text {Newt }}} \\
\end{array}$ \\
\hline 0.0 & 1.0000000 & 1.1692906 & 1.0000 & 1.5673 & 1.000 & 1.7678 & 1.000 & 2.027 & 1.000 & 2.376 \\
\hline 0.5 & 1.0000000 & 1.0214102 & 1.0000 & 1.1507 & 1.000 & 1.2325 & 1.000 & 1.345 & 1.000 & 1.505 \\
\hline 1.0 & $\mathbf{0 . 9 5 5} 5952$ & $\mathbf{0 . 9 2 5} 1084$ & 0.7225 & -0.8648 & 0.939 & -7.8434 & 0.456 & 16.01 & 1.091 & 8.443 \\
\hline 1.5 & $\mathbf{0 . 9 6 8} 1616$ & $\mathbf{0 . 9 6 8} 6094$ & 0.9188 & $\mathbf{0 . 9 0 7 4}$ & 0.940 & 0.9069 & 0.995 & 0.924 & 1.094 & 0.967 \\
\hline 2.0 & 0.9681512 & 0.9676191 & 0.9184 & 0.8850 & 0.939 & 0.8671 & 0.993 & 0.860 & 1.091 & 0.867 \\
\hline 2.5 & 0.9675775 & $\mathbf{0 . 9 6 7 6 9 8 1}$ & 0.8624 & $\mathbf{0 . 8 8 9 0}$ & 0.799 & 0.8754 & 0.692 & 0.875 & 0.504 & 0.893 \\
\hline 3.0 & 0.9677265 & 0.9677247 & 0.8951 & 0.8914 & 0.895 & 0.8804 & 0.928 & 0.883 & 1.022 & 0.903 \\
\hline 3.5 & 0.9677274 & 0.9677233 & 0.8957 & 0.8912 & 0.897 & 0.8798 & 0.934 & 0.882 & 1.036 & 0.900 \\
\hline
\end{tabular}

waveform at a significant level. Nevertheless, the values derived in this section may not be optimal. Thus, we emphasize that the results of this section constitute merely an exercise demonstrating the feasibility of adjusting the PN parameters to optimize the agreement of the PN flux function with numerical data.

The least-squares fits are done on $F(\varpi) / F_{\text {Newt }}(\varpi)$ [see Eq. (73)]. In the case of $\mathrm{T}$ approximants, we fit for the unknown 4PN-order coefficient in Eq. (19) for the equalmass case. We perform a least-squares fit of the $4 \mathrm{PN}$-order function $\mathcal{F}_{8}(\nu=1 / 4)=A_{8}+B_{8} \log v_{\Omega}$ over the orbitalfrequency range $M \Omega=0.02-0.08$ which starts after the first $9 \mathrm{GW}$ cycles. We obtain $A_{8}=-141, B_{8}=102$. We notice that when we perform the fit over the first 15 (or 20) GW cycles, spanning the frequency region $M \Omega=$

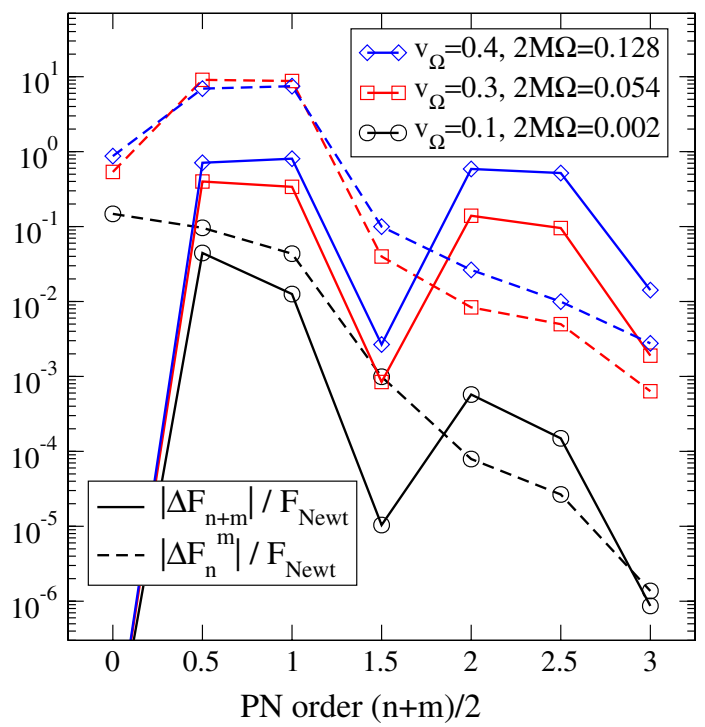

FIG. 10 (color online). Cauchy convergence test of $F / F_{\text {Newt }}$ for $\mathrm{T}$ and $\mathrm{P}$ approximants. We plot $\Delta F_{n+m} \equiv F_{n+m+1}-F_{n+m}$, and $\Delta F_{n}^{m} \equiv F_{n+1}^{m}-F_{n}^{m}$ for different values of $v_{\Omega}$. The $\mathrm{T}$ and $\mathrm{P}$ approximants are given by Eqs. (19) and (39), respectively. Note that the $\mathrm{P}$ approximant has an extraneous pole at 1PN order at $v_{\Omega}=0.326$. We use $v_{\text {lso }}=v_{\text {lso }}^{2 \mathrm{PN}}$, and $v_{\text {pole }}=v_{\text {pole }}^{2 \mathrm{PN}}$.
$0.0168-0.0235(M \Omega=0.0168-0.0283)$, the agreement becomes worse. The resulting flux is shown in Fig. 11. The relative difference with the numerical flux is at most $\sim 0.8 \%$.

We repeat this analysis in the case of $\mathrm{P}$ approximants. Because the latter also depend upon $v_{\text {pole }}$, we perform two least-squares fits. In the first fit, we fix $v_{\text {pole }}$ to the value given by Eq. (41) and apply the least-squares fit to $\mathcal{F}_{8}(\nu=$ 1/4) obtaining $A_{8}=-1382, B_{8}=197$.

In the second fit, we vary $v_{\text {pole }}$. When varying $v_{\text {pole }}$ in the $\mathrm{P}$ approximant at 3.5PN order, extraneous poles appear at low values of $v_{\Omega}$. Therefore, in order to push these poles to very high frequency, we follow the suggestion of Ref. [18], and use $\mathrm{P}$ approximants at $4 \mathrm{PN}$ order, where the 4PN coefficient is set to its known value in the test-mass limit. Furthermore, the logarithm in the flux is not factored out,

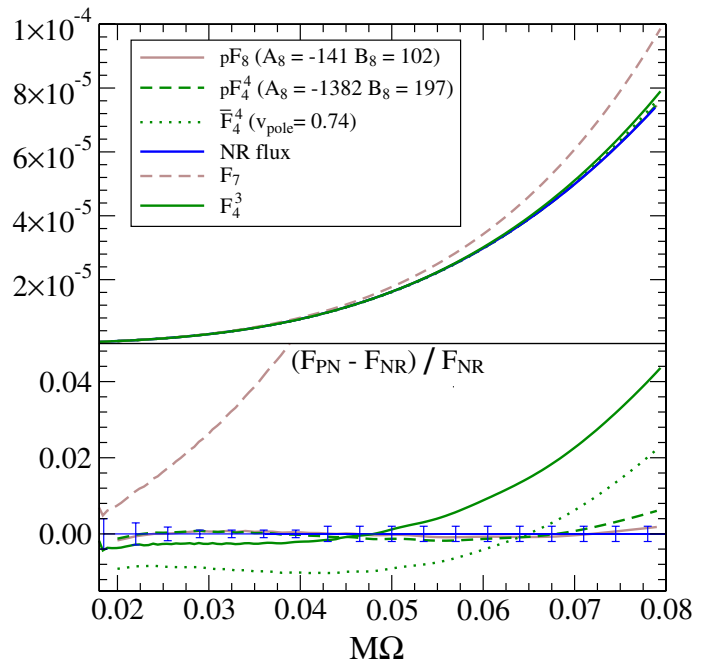

FIG. 11 (color online). Fitting several PN approximants to the numerical flux. The $x$ axis denote the orbital frequency $\Omega$. Because the numerical flux is computed as function of the GW frequency, we use for the numerical flux $\Omega \equiv \varpi / 2$. The blue bars indicate estimated errors on the numerical flux; see Fig. 2. For notation see Table I and caption therein. 
but treated as a constant when Padé summation is done. This cure may fail for different mass ratios if new extraneous poles appear at low frequency. The least-squares fit gives $v_{\text {pole }}=0.74$. All the results for the $\mathrm{P}$ approximants are displayed in Fig. 11, where we also show the T and $\mathrm{P}$ approximants at 3.5PN order without any fit.

Figure 11 might suggest that by introducing higherorder PN coefficients in the flux, the numerical flux can be fit better by $\mathrm{T}$ approximants than by $\mathrm{P}$ approximants. However, this result may depend on the use of orbital frequency instead of GW frequency. In Sec. VIC (see Fig. 18), we employ the fit values obtained in this study and show phase differences between NR and tuned EOB models.

Finally, we attempted to extract PN coefficients higher than 3.5PN order from the numerical flux, as was done at 2PN, 2.5PN, and 3PN order in Ref. [50] in the test-mass limit. Unfortunately, the differences between numerical flux and $\mathrm{T}$ approximants are so large- even at the beginning of the numerical waveform - that we were not able to extract even known PN coefficients, like the ones at 3PN and 3.5PN order. Thus, to fit unknown PN coefficients would require a numerical simulation with more cycles starting at lower frequency.

\section{ESTIMATION OF THE (DERIVATIVE OF THE) CENTER-OF-MASS ENERGY}

In the previous section, we analyzed and compared PN and numerical energy fluxes. The energy of the binary is the second fundamental ingredient in the construction of adiabatic PN approximants. Unfortunately, there is no way to extract the energy for the numerical simulation as a function of a gauge-invariant quantity such as the GW frequency, so that it is impossible to compare PN and NR energies directly. The frequency derivative $\dot{\varpi}$, however, is easily accessible in the numerical data, and, in the adiabatic approximation is intimately related to the energy, as can be seen by rewriting the energy-balance equation (12), in the form

$$
\frac{d \varpi}{d t}=-\frac{F}{d E / d \varpi}
$$

Therefore, we begin this section with a comparison between numerical $\dot{\varpi}$ and the predictions of various PN approximants. For the PN approximants, we compute $h_{22}$ as usual (i.e., using energy balance to compute the orbitalfrequency derivative $\dot{\Omega}$ ), and take a time derivative to obtain $\dot{h}_{22}$ and extract $\dot{\varpi}$ from it. The waveform $h_{22}$ for the E approximants is computed using Eqs. (42) and (44)(46) in Sec. III C. Figure 12 plots the numerical $\dot{\varpi}$ and its value for $\mathrm{T}, \mathrm{P}$, and also $\mathrm{E}$ approximants at 3.5PN order.

In order to emphasize differences between the different $\dot{\varpi}$, we normalize the data in Fig. 12 by the Newtonian value of $\dot{\varpi}$,

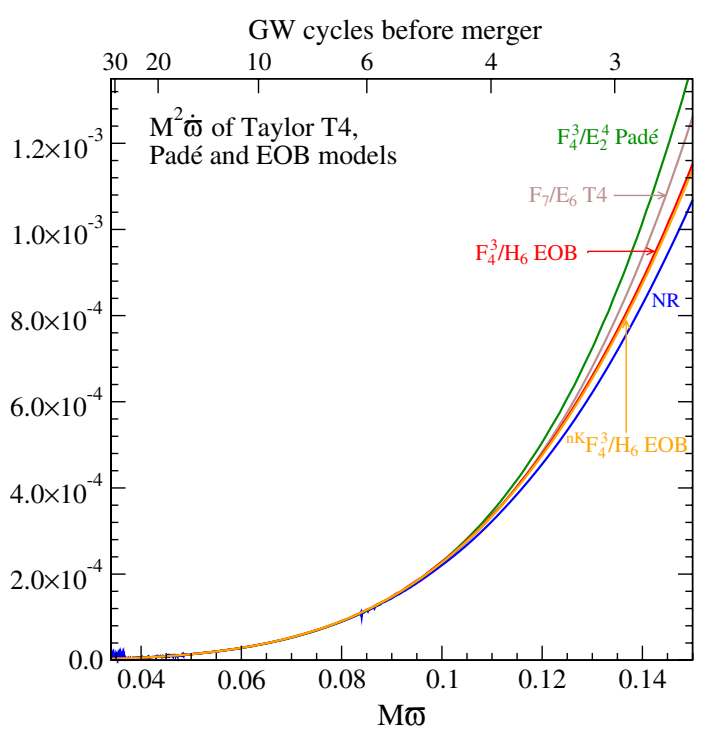

FIG. 12 (color online). GW frequency derivative $\dot{\varpi}$ for the numerical relativity simulation and various $\mathrm{PN}$ approximants at 3.5PN order. For notation see Table I and caption therein.

$$
\frac{\dot{\varpi}}{\dot{\varpi}_{\text {Newt }}} \equiv \frac{\dot{\varpi}}{\frac{192}{5} \frac{\nu}{M^{2}}\left(\frac{M \varpi}{2}\right)^{11 / 3}}
$$

The normalization is used only to eliminate the leadingorder behavior of the various curves in Fig. 12; therefore, to compute the denominator of Eq. (75) we have simply substituted $\varpi / 2$ rather than $\Omega$ into the Newtonian formula for the frequency derivative.

The normalized frequency derivatives are shown in Fig. 13. At low frequencies, $\dot{\varpi}$ is very challenging to compute in numerical simulations, resulting in comparatively large numerical uncertainties. Therefore, for frequencies $M \varpi \lesssim 0.045$ we can merely conclude that PN and NR are consistent with each other (i.e., are within the numerical error bars of about $10 \%$ ).

The 3.5PN Taylor T4 model (labeled $F_{7} / E_{6} T 4$ ) agrees very well with the numerical simulation up to $M \varpi \approx 0.1$; this observation is consistent with the excellent agreement between TaylorT4 (3.5PN) and the numerical simulation observed in Boyle et al. [12], who compared up to this frequency. Beyond $m \varpi=0.1$, however, $\dot{\varpi} / \dot{\varpi}_{\text {Newt }}$ for Taylor T4 continues to increase (as for all other Taylor and Padé models considered here), whereas for the numerical simulation, $\dot{\varpi} / \dot{\varpi}_{\text {Newt }}$ flattens (this behavior was also observed in Ref. [18]). Only the E approximants at 3.5PN order reproduce the flattening of $\dot{\varpi} / \dot{\varpi}_{\text {Newt }}$ at high frequencies, with the closest being the one which uses the nonKeplerian Padé flux $\left({ }^{\mathrm{nK}} F_{4}^{3}\right.$ ). Because the frequency derivative is the relevant quantity that determines the phase evolution, the turning over of $\dot{\varpi} / \dot{\varpi}_{\text {Newt }}$ for the nonadiabatic models in Fig. 13 suggests that, at high frequency, nonadiabatic analytical models might be superior to adiabatic models. 


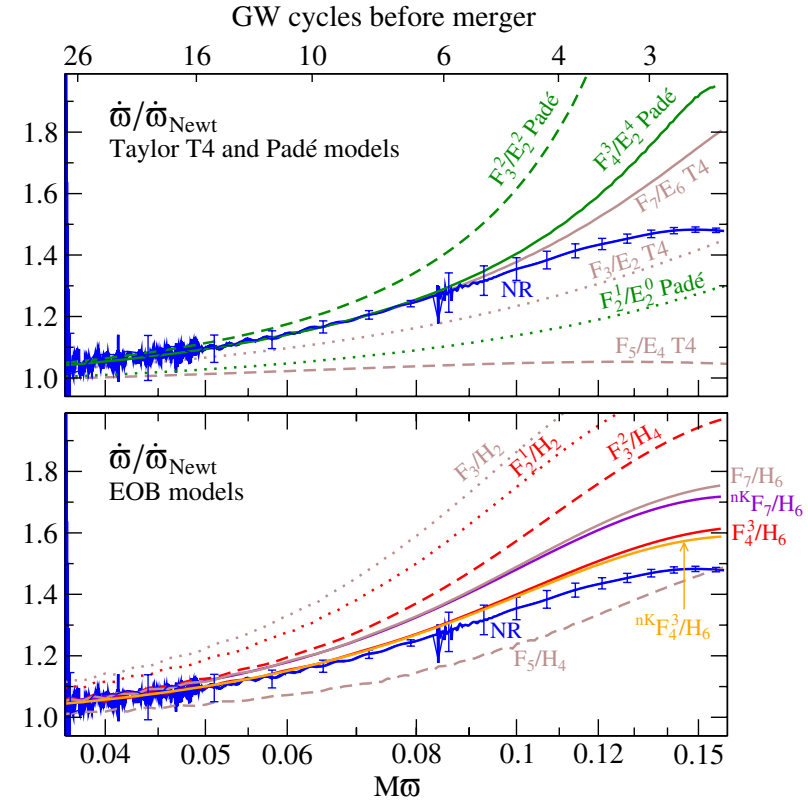

FIG. 13 (color online). Comparison of $\dot{\varpi}$ for the numerical results and various PN approximants. Dotted, dashed, and solid lines correspond to 1.5PN, 2.5PN, and 3.5PN models, respectively. For notation see Table I and caption therein.

If sufficient smoothing is applied to the numerical $\dot{\varpi}$ it becomes a smooth curve even at low frequencies. Figure 14 presents a comparison between such a heavily smoothed numerical curve and the PN approximants. As already pointed out, all PN approximants are consistent to within

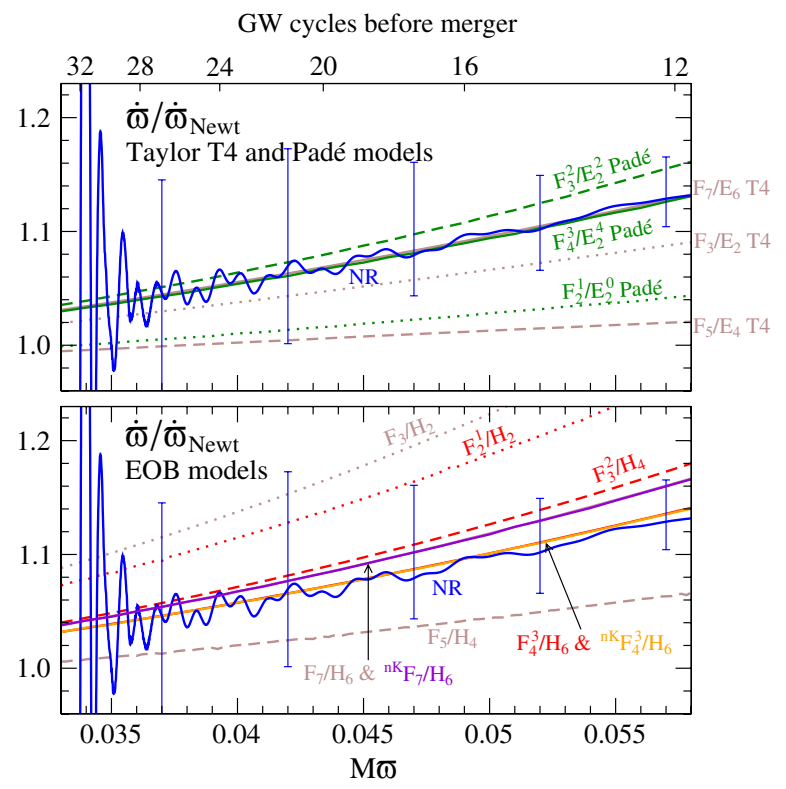

FIG. 14 (color online). Comparison of PN $\dot{\varpi}$ with a heavily smoothed version of the numerical $\dot{\varpi}$. Solid lines represent 3.5PN models and NR; dashed and dotted lines correspond to 3PN and 2.5PN models, respectively. For notation see Table I and caption therein. our estimated numerical errors at low frequencies. However, the NR result in Fig. 14 is notably closer to the 3.5PN approximants than to lower order PN approximants. This good agreement provides a further validation of the numerical code used in Boyle et al. [12]. It also indicates that our error analysis in Sec. II may be overly conservative.

Our comparisons of $\dot{\varpi}$ reveal a lot of information about the PN approximants. However, $\dot{\varpi}$ depends on both flux and energy [see Eq. (74)], and so these comparisons do not yield information about flux or energy separately. To isolate effects due to the PN energy, we rearrange Eq. (74) further, such that it yields in the adiabatic approximation the derivative of the center-of-mass energy for the numerical simulation:

$$
\left[\frac{d E}{d \varpi}\right]_{\mathrm{NR}}=-\frac{F_{\mathrm{NR}}}{[d \varpi / d t]_{\mathrm{NR}}}
$$

The relative error in $[d E / d \varpi]_{\mathrm{NR}}$ is obtained as the rootsquare-sum of the relative errors of flux and frequency derivative (see Figs. 2 and 4). In Fig. 15 we compare the latter with T, P, and E approximants. For adiabatic T4 and Padé models, we compute $d E / d \varpi$ by taking derivatives of $E(\Omega)$ in Eq. (14) with respect to $\Omega$ and then expressing the derivative in terms of $\varpi(\Omega)$. For nonadiabatic EOB models, we compute $d E / d \varpi$ from the ratio of $F_{\mathrm{PN}}$ and $[d \varpi / d t]_{\mathrm{PN}}$ as obtained from Figs. 7 and 12. The closeness between the numerical result and adiabatic PN approximants is expected only in the range of frequencies over which the balance equation and the adiabatic approxima-

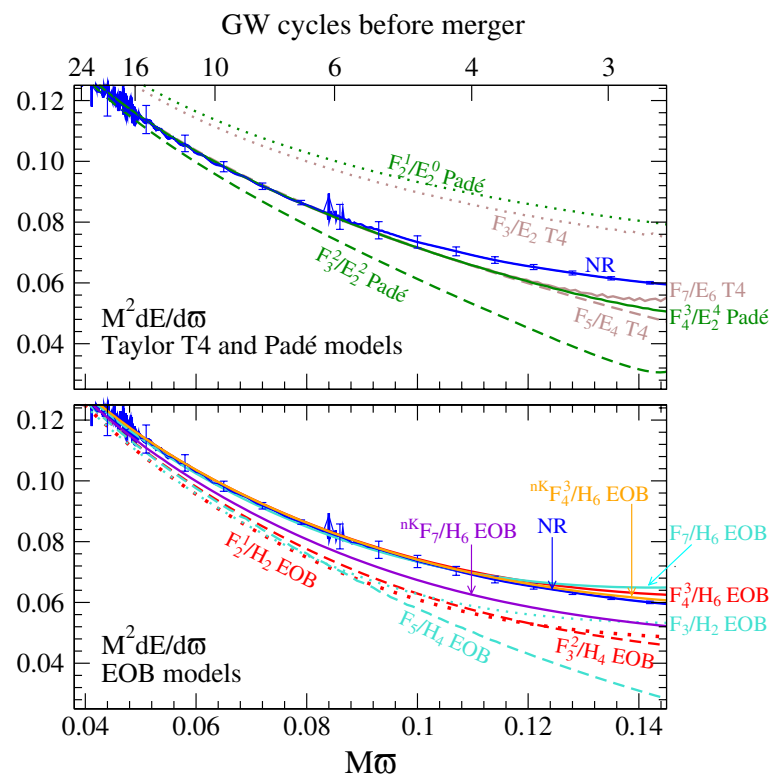

FIG. 15 (color online). We compare $d E / d \varpi$ versus GW frequency $\varpi$ for numerical relativity [see Eq. (76)] and PN approximants. Solid lines represent 3.5PN models and NR; dashed and dotted lines correspond to 3PN and 2.5PN models, respectively. For notation see Table I and caption therein. 
tion are valid. The upper panel of Fig. 15 shows the Taylor and Padé adiabatic models. The plot suggests that around $M \varpi \sim 0.08$ nonadiabatic effects are no longer negligible. At lower frequencies, both 3.5PN order adiabatic approximants (Padé and Taylor T4) match the numerical result very well. Taylor T4 at $2.5 \mathrm{PN}$ matches well, too, although its frequency derivative $\dot{\varpi}$ and flux differ significantly from NR (see Figs. 9 and 13). The T approximant at 3.5PN order is closest to the numerical result. The lower panel of Fig. 15 shows the nonadiabatic E approximants. We notice that the nonadiabatic models, especially at 3.5PN order, follow quite nicely the behavior of the numerical derivative of the center-of-mass energy. The E approximant with nonKeplerian flux is closest to the numerical result. This analysis emphasizes again the relevance of including nonadiabatic effects in the analytical model [23].

\section{COMPARING WAVEFORMS}

Here we compare the numerical waveform to various PN waveforms, basically extending the analysis of Boyle et al. [12] to include Padé and EOB waveforms. Because the (2, 2) mode dominates the waveform for an equal-mass nonspinning binary, we restrict the comparison to only this mode. As in [12], we use $\Psi_{4}^{22}$ and the GW phase and frequency $\omega$ defined by Eq. (4) when comparing waveforms.

For the comparisons presented in this section, the uncertainty in the phase of the numerical waveform is roughly 0.02 radians. This number includes numerical errors (e.g., due to convergence and extrapolation of the waveform to infinite extraction radius), as well as modelling errors due to slightly nonzero eccentricity and spin of the numerical simulation; see Ref. [12], Sec. IV for details. We note that the modelling errors have decreased since the analysis in Ref. [12] because the new matching procedure reduces the impact of eccentricity, and because the more sophisticated spin diagnostics presented in Ref. [55] resulted in a smaller bound on the residual spin.

\section{A. Matching procedure}

Each PN waveform has an arbitrary time offset $t_{0}$ and phase offset $\phi_{0}$ with respect to the NR waveform. The procedure used by Boyle et al. [12]—as well as in various other papers before it, such as $[10,11]$ —sets these constants by ensuring that the GW phase and frequency match at a fiducial time. Unfortunately, when matching at low frequency this method is sensitive to noise and to residual eccentricity in the numerical waveform, and does not easily translate into a robust and automatic algorithm. Since we want to match as early as possible (where we expect the PN approximants to be valid), we propose to use, instead, a matching procedure which achieves the same goal, but extends over a range of data. This procedure is similar to the one proposed by Ajith et al. [17], but whereas we match only the GW phase, Ajith et al. match the entire gravitational waveform-including the amplitude-and include an overall amplitude scaling. This method can be easily implemented as a fairly automatic algorithm, robust against noise and residual eccentricity.

Using the phase of the numerical and PN waveforms, we define the quantity

$$
\Xi(\Delta t, \Delta \phi)=\int_{t_{1}}^{t_{2}}\left[\phi_{\mathrm{NR}}(t)-\phi_{\mathrm{PN}}(t-\Delta t)-\Delta \phi\right]^{2} d t .
$$

Here, $t_{1}$ and $t_{2}$ represent the chosen range over which to compare. Minimizing this quantity by varying the time and phase offsets $\Delta t$ and $\Delta \phi$ produces the optimal values for these quantities in a least-squares sense. Then to compare PN and NR waveforms, we compare the (unchanged) NR waveform with an offset PN waveform defined by

$$
\Psi_{4, \mathrm{PN}}(t)=A_{\mathrm{PN}}(t+\Delta t) \mathrm{e}^{-i\left[\phi_{\mathrm{PN}}(t+\Delta t)+\Delta \phi\right]} .
$$

With reasonable first guesses for $\Delta t$ and $\Delta \phi$, the function $\Xi$ is quite nicely paraboloidal. Thus, even simple minimization routines work well. However, in cases where speed is an issue, the problem can be reduced to one dimension. For a given value of $\Delta t$, the optimization over $\Delta \phi$ may be done analytically by setting

$$
\Delta \phi(\Delta t)=\frac{\int_{t_{1}}^{t_{2}}\left[\phi_{\mathrm{NR}}(t)-\phi_{\mathrm{PN}}(t-\Delta t)\right] d t}{t_{2}-t_{1}}
$$

Using this value of $\Delta \phi$ for a given value of $\Delta t$ decreases the number of function evaluations needed to find the minimum. This can be very useful for large data sets, or situations where many such matches need to be done.

The choice of $t_{1}$ and $t_{2}$ involves some degree of judgment. Preferably, $t_{1}$ should be as early as possible, while not being contaminated by junk radiation. We choose $t_{1}=$ $1100 M$, corresponding to $M \omega=0.037$. Similarly, $t_{2}$ should be as early as possible, but far enough from $t_{1}$ so that the integration averages over the noise. In addition, the effects of the small but nonzero orbital eccentricity show up as oscillations in the phase, as can be seen, for example, in the range $t \in[1100,1900] M$ in Fig. 17. We would like $t_{2}$ to be large enough so that the integration averages over several cycles of this oscillation, thus resulting in less bias due to eccentricity. Here we use $t_{2}=1900 M$, corresponding to $M \omega=0.042$. We have checked that changing the values of $t_{1}$ and $t_{2}$ by $\pm 100 M$ changes the resulting phases by less than a few thousandths of a radian through the end of the numerical waveform.

This method is quite similar to the one suggested in Ref. [17]. However, here, we consider only the phase and not the amplitude of the waveform. Because we restrict the analysis only to the $(2,2)$ waveform mode of an equalmass binary and compare only the phase and not the 
amplitude, we think it is reasonable to have neglected the amplitude in the matching procedure.

\section{B. Padé waveforms}

In Fig. 16 we plot the phase difference between the numerical, $\mathrm{T}$ and $\mathrm{P}$ approximants [21,24,32] at the times when the numerical waveform reaches $\mathrm{GW}$ frequencies $M \omega=0.063$ and $M \omega=0.1$. The phase differences are plotted versus the PN order. The phase difference at $M \omega=$ 0.1 of the $\mathrm{P}$ approximant at $3.5 \mathrm{PN}$ order is -0.12 radians. When comparing with generic Taylor approximants, we notice that the phase differences of the $\mathrm{P}$ approximants are less scattered as the PN order is increased. This might be due to the fact that $\mathrm{P}$ approximants of the energy flux are closer to the NR flux, especially for lower $v_{\Omega}$ where the phase accumulates the most. Figure 16 could be contrasted with Tables III and IV of Ref. [21] which show the overlaps between the numerical waveform and $\mathrm{P}$ approximants at subsequent PN orders, in the test-mass limit case. The behavior of the $\mathrm{P}$ approximants in Fig. 16 are consistent with the behavior of $\dot{\varpi}$ seen in Fig. 13: At 1.5PN, Padé has smaller $\dot{\varpi}$ than the numerical simulation, at 2.5PN, Padé has larger $\dot{\varpi}$. Consequently, $\Phi_{\mathrm{PN}}-\Phi_{\mathrm{NR}}$ is negative at 1.5PN order and positive at 2.5PN order. For 3.5PN order, the $\mathrm{P}$ approximant in Fig. 13 agrees very well with the numerical simulation (at least for $M \varpi \lesssim 0.1$ ), which translates into excellent agreement in Fig. 16.

In Fig. 17, we explore the possibility of reducing the phase differences between the numerical waveform and $\mathrm{P}$

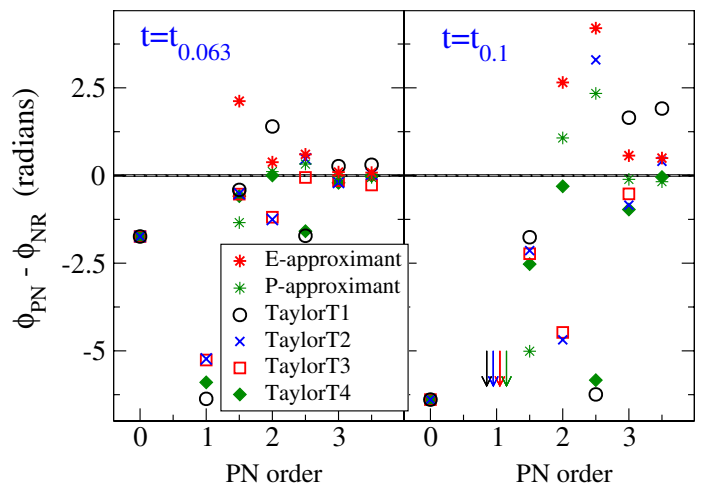

FIG. 16 (color online). Phase differences between the numerical waveform, and untuned, original EOB, untuned Padé, and Taylor waveforms, at two selected times close to merger. The E approximants are $F_{n}^{m} / H_{p}$, while the $\mathrm{P}$ approximants are $F_{n}^{m} / E_{p}^{q}$ (see Table I and caption therein). Waveforms are matched with the procedure described in Sec. VI A and phase differences are computed at the time when the numerical simulation reaches $M \omega=0.063$ (left panel) and $M \omega=0.1$ (right panel). Differences are plotted versus PN order. Note that at $1 \mathrm{PN}$ order the Padé flux has an extraneous pole at $v=0.326$ causing a very large phase difference. The thick black line indicates the uncertainty of the comparison as discussed in Sec. VI, $\mid \Phi_{\mathrm{PN}}-$ $\Phi_{\mathrm{NR}} \mid \leq 0.02$ radians.

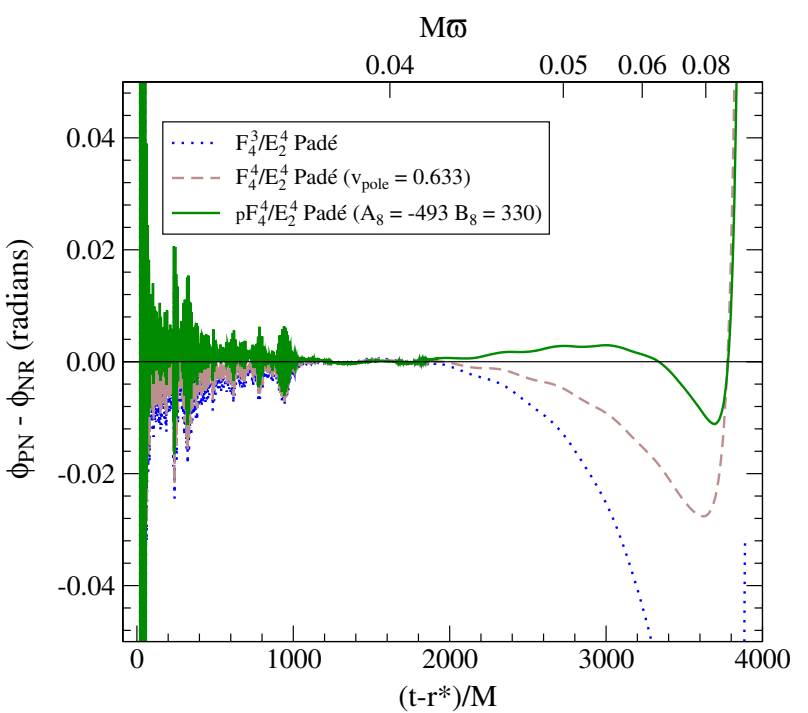

FIG. 17 (color online). Phase differences between untuned and tuned $\mathrm{P}$ approximants and $\mathrm{NR}$. The untuned $\mathrm{P}$ approximant is $F_{4}^{3} / E_{2}^{4}\left(v_{\text {lso }}=v_{\text {lso }}^{2 \mathrm{PN}}, v_{\text {pole }}=v_{\text {pole }}^{2 \mathrm{PN}}\right)$. The tuned $\mathrm{P}$ approximants are $F_{4}^{4} / E_{2}^{4}$ and tunable $v_{\text {pole }}\left(v_{\text {lso }}=v_{\text {lso }}^{2 \mathrm{PN}}\right)$ and $p F_{4}^{4} / E_{2}^{4}\left(v_{\text {lso }}=\right.$ $v_{\text {lso }}^{2 \mathrm{PN}}, v_{\text {pole }}=v_{\text {pole }}^{2 \mathrm{PN}}$ ) with tunable $A_{8}$ and $B_{8}$. In all cases, waveforms are matched over $t-r^{*} \in[1100,1900] M$.

approximants by (i) varying $v_{\text {pole }}$ or (ii) introducing the pseudo 4PN order coefficient $\mathcal{F}_{8}(\nu=1 / 4)=A_{8}+$ $B_{8} \log v_{\Omega}$ in the energy flux. We tune the coefficients by minimizing the sum of the squares of the phase difference at $t_{0.063}$ and $t_{0.1}$. We find that if $v_{\text {pole }}=0.633$, the $\mathrm{P}$ approximant $F_{4}^{4} / E_{2}^{4}$ has a maximum phase difference before $M \omega=0.1$ smaller than the numerical error in the simulation. A similar result is obtained for the $\mathrm{P}$ approximant $p F_{4}^{4} / E_{2}^{4}$ if we use $v_{\text {pole }}=v_{\text {pole }}^{2 \mathrm{PN}}=0.6907$, and tune $A_{8}=-493, B_{8}=330$.

\section{Effective-one-body waveforms}

In Fig. 16, we also plot the phase differences between the numerical and the untuned, original $\mathrm{E}$ approximants [22-24] $F_{n}^{m} / H_{p}$. At 3.5PN order the phase difference at $M \omega=0.1$ is 0.50 radians. We also computed the phase differences at $M \omega=0.1$ of the E approximants ${ }^{{ }^{n K}} F_{4}^{3} / H_{7}$, ${ }^{\mathrm{nK}} F_{7} / H_{7}$, and $F_{7} / H_{7}$, and found $0.45,2.56$, and 2.7 radians, respectively. Thus, for untuned EOB models it is crucial to have introduced the Padé flux. When contrasting the original E approximants with generic Taylor approximants, we find that the phase differences are less scattered as the PN order is increased. However, despite the fact that the Padé-based EOB flux is closer to the numerical flux (see Figs. 8 and 9), untuned, original $\mathrm{E}$ approximants accumulate more phase difference than $\mathrm{P}$ approximants. This could be a consequence of the fact that independently of the flux and the energy functions, what seems to matter is the way the equations of motions are solved to get the phasing. 
Because of the reduction of the dynamics to a few crucial functions determining the inspiral evolution $[22,23,25]$, notably $A, D$, and $\mathcal{F}$, and because of the rather simple procedure to match the inspiral(-plunge) waveform to the ringdown waveform, the EOB model turned out to be particularly suitable for matching the full numerical waveforms $[9,16,18,20,27]$. In view of a future study which will include merger and ringdown, we start here exploring the possibility of improving the agreement with numerical waveforms by tuning the pseudo 4PN order coefficients $a_{5}, A_{8}$, and $B_{8}$ and/or, if present, the pole location $v_{\text {pole }}$. In the lower panel of Fig. 18, using different $v_{\text {pole }}$ values, we show the phase differences computed at $t_{0.063}$ and $t_{0.1}$ as functions of the unknown PN-expansion coefficient $a_{5}$ [see Eq. (49)]. As first pointed out and discussed in Ref. [18] (see e.g., Fig. 3 therein), we find that there is a strong degeneracy between $a_{5}$ and $v_{\text {pole. }}$. In fact, for different $v_{\text {pole }}$
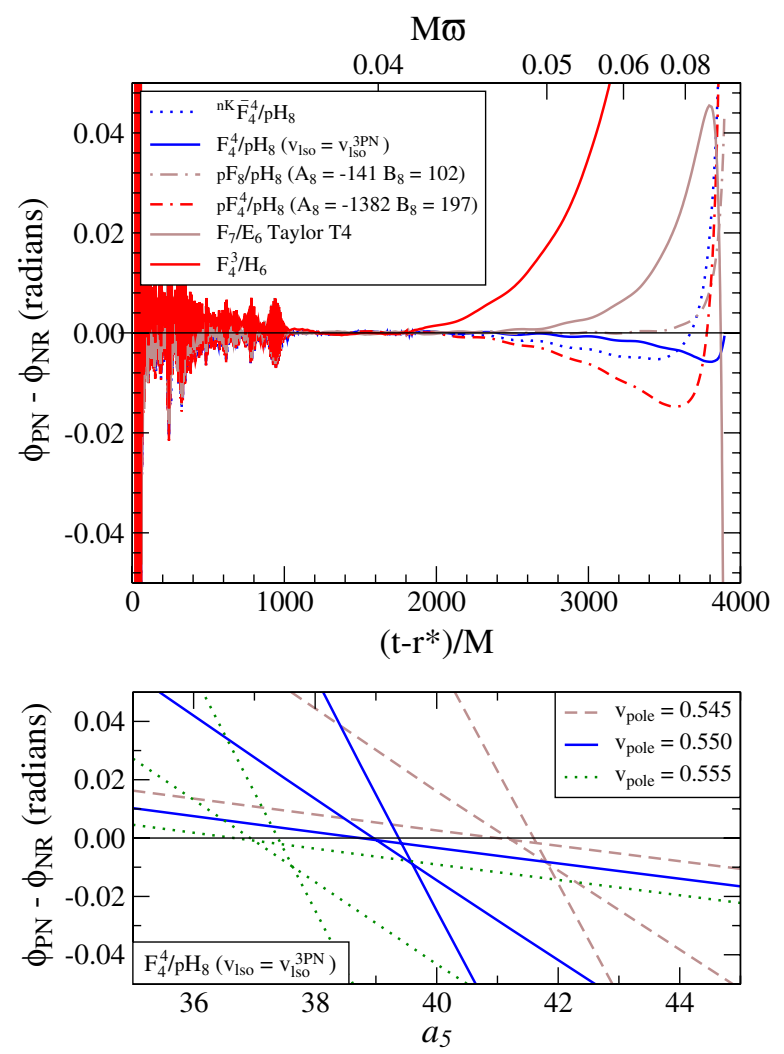

FIG. 18 (color online). The upper panel shows phase differences versus time (lower $x$ axis) and versus GW frequency (upper $x$ axis) for several tuned and untuned $\mathrm{E}$ approximants. For the tuned models, the optimal $a_{5}$ and $v_{\text {pole }}$ values displayed in Table III. In the lower panel, we show phase differences between numerical and $\mathrm{E}$ approximants computed at $t_{0.063}, t_{0.1}$, and the end of the numerical simulation $t_{0.16}$, as functions of $a_{5}$. For the same color and style, the curve with the steepest slope corresponds to $t_{0.16}$ and the curve with the smallest slope corresponds to $t_{0.063}$. (For notation see Table I and caption therein.) values, the curves in Fig. 18 are almost identical except for a shift in $a_{5}$. Although in this test we use the E approximant $F_{4}^{4} / p H_{8}\left(v_{\text {lso }}=v_{\text {lso }}^{2 \mathrm{PN}}\right)$, we find that this degeneracy appears in all $\mathrm{E}$ approximants considered.

To obtain the optimal $a_{5}$ and $v_{\text {pole }}$ that minimize phase differences during the entire numerical simulation, we first choose an arbitrary $v_{\text {pole }}$ in the range of degeneracy. Then, we determine the $a_{5}$ value by minimizing the sum of the squares of the phase difference at $t_{0.063}$ and $t_{0.1}$. In the upper panel of Fig. 18, we show phase differences in time and GW frequency for several $\mathrm{E}$ approximants using those optimal $a_{5}$ and $v_{\text {pole }}$ values, which are given in Table III. In Fig. 18, we also show phase differences for $E$ approximants with pseudo 4PN order coefficients determined by the flux fit of Sec. IV B (see Fig. 11) and tunable $a_{5}$. The optimal $a_{5}$ values are shown in Table III. The smaller phase differences along the entire inspiral are obtained with the $\mathrm{E}$ approximants with Padé flux $F_{4}^{4} / p H_{8}\left(v_{\text {lso }}=v_{\text {lso }}^{2 \mathrm{PN}}\right)$ and tunable $v_{\text {pole }}, a_{5}$ and Taylor flux $p F_{8} / p H_{8}$ with tunable $A_{8}$, $B_{8}, a_{5}$. We notice that for $t>t_{0.1}$ the phase difference increases more abruptly for the latter model. In the best case, the absolute phase difference during the entire numerical simulation is within the numerical error, i.e., within 0.02 radians. The choice of the best tuned E approximant $[15,16,18-20]$ will be determined once merger and ringdown are included, and when long and accurate comparisons with numerical simulations are extended to BBH with mass ratio different from one.

Finally, in Ref. [18], Damour and Nagar extracted the data of the numerical simulation used in the present paper from one of the figures of Ref. [12] and compared those data with the EOB approach. They found for their "nontuned" EOB model phase differences \pm 0.05 radians. This phase difference is smaller than the phase differences we discuss in this paper for untuned EOB models (see Fig. 16 and discussion around it). However, we notice that \pm 0.05 radians in Ref. [18] refers to half the maximum phase difference accumulated over the entire evolution when matching the numerical and EOB phases at $M \omega=0.1$. By contrast, in this paper, and, in particular, in Fig. 16, we match numerical and EOB phases in a time interval and compute the phase differences at $M \omega=$ 0.1 .

Moreover, we observe that their "nontuned" EOB model is not really untuned, because it uses the Padé summation of the radial potential at 4PN order and then sets $a_{5}=0$. This is not equivalent to using the radial potential at 3.5PN order with $a_{5}=0$. In fact, to recover the 3.5PN order Padé radial potential from the 4PN order Padé potential one should use $a_{5}=-17.16$. They also use the non-Keplerian flux at $4 \mathrm{PN}$ order ${ }^{\mathrm{nK}} \bar{F}_{4}^{4}$ which is different from the 3.5PN order one ${ }^{\mathrm{nK}} F_{4}^{3}$. For our untuned EOB model at 3.5PN order which uses ${ }^{{ }^{n K}} F_{4}^{3}$ and the EOB dynamics at 3PN order, if we apply Ref. [18] procedure and compute half the maximum phase difference when 
TABLE III. Optimal $a_{5}$ and $v_{\text {pole }}$ that minimize phase differences between tuned EOB models and the numerical simulation.

\begin{tabular}{lcrc}
\hline \hline EOB model and fixed parameters & \multicolumn{1}{c}{$a_{5}$} & $v_{\text {pole }}$ \\
\hline $\mathrm{nK}_{\bar{F}}^{4} / p H_{8}$ & - & 29.78 & 0.52 \\
$F_{4}^{4} / p H_{8}$ & $v_{\text {lso }}=v_{\text {lso }}^{2 \text { PN }}$ & 39.35 & 0.55 \\
$p F_{8} / p H_{8}$ & $A_{8}=-141, B_{8}=102$ & 5.32 & N/A \\
$p F_{4}^{4} / p H_{8}$ & $A_{8}=-1382, B_{8}=197, v_{\text {lso }}=v_{\text {lso }}^{2 \text { 2N }}, v_{\text {pole }}=v_{\text {pole }}^{2 \text { PN }}$ & -3.10 & N/A \\
\hline \hline
\end{tabular}

matching the numerical and EOB phases at $M \omega=0.1$, we find a phase difference of \pm 0.18 radians.

\section{CONCLUSIONS}

In this paper, using a highly accurate and long numerical simulation [12] of a nonspinning equal-mass black-hole binary, we compute the gravitational waveform, GW energy flux, and GW frequency derivative. Imposing the balance equation, we also estimate the (derivative of) center-of-mass energy. We compare these quantities to those computed using adiabatic TaylorT4 and Padé $[21,24,32]$, and nonadiabatic EOB PN approximants [2224].

We find that for the first $15 \mathrm{GW}$ cycles, the 3.5PN order $\mathrm{T}$ approximant and the 3.5PN order untuned $\mathrm{P}$ and $\mathrm{E}$ approximants (see Table I) reproduce the numerical results for energy flux, GW frequency derivative, and (derivative of) center-of-mass energy quite well (see Figs. 8, 9, and 13-15), but with interesting differences.

We attempted to study the convergence of the PN expansion for the energy flux. ${ }^{9}$ We find that Padé approximants to the flux introduced in Ref. [21] do not accelerate the convergence of the Taylor series, but are closer to the numerical flux than are the T approximants. In particular, the Taylor flux at all orders through $3.5 \mathrm{PN}$ is outside the numerical flux error bars even $\sim 25 \mathrm{GW}$ cycles before merger (see Fig. 8). We find that the nonadiabatic nonKeplerian E approximants to the flux at 3.5PN order are within $\sim 2 \%$ of the numerical flux over the entire frequency range we consider (see Fig. 9). The disagreement of the Taylor flux demonstrates that the good phase agreement of TaylorT4 is indeed accidental. For the E approximants, the much better agreement of flux and energy would suggest that the good agreement of the final EOB phase might be not accidental, and that we might expect that the performance of the E approximants could be more robust to changes in mass ratio and spin.

Quite interestingly, in the equal-mass case the numerical normalized energy flux $F / F_{\text {Newt }}$ starts decreasing at high frequency during the late part of the inspiral and blurred

\footnotetext{
${ }^{9} \mathrm{We}$ also tried to apply the criterion suggested in Ref. [56] to assess the region of validity of the PN series for the flux in the equal-mass case. Unfortunately, the numerical simulation starts at too high a frequency, when the Taylor series at 3.5PN order seems to already be outside the region of validity.
}

plunge (see Fig. 9). This differs from the behavior of $F / F_{\text {Newt }}$ in the test-mass limit (see Fig. 19). Both the Taylor and Padé-based E approximants with nonKeplerian flux [34] show a similar decreasing behavior at high frequency. This fact suggests that if a pole is present in the energy flux of equal-mass binaries, it is located at a larger frequency than that at which the common apparent horizon forms. As seen in Sec. IV B, when fitting for $v_{\text {pole }}$ we obtain $v_{\text {pole }}(\nu=1 / 4)=0.74$, which is to be contrasted with the test-mass case $v_{\text {pole }}(\nu=0)=1 / \sqrt{3} \approx 0.58$. These values of $v_{\text {pole }}$ correspond to orbital frequencies $M \Omega=0.405$ and $M \Omega=0.192$, respectively.

For the GW frequency derivative $\dot{\varpi}$, we find that at low frequency the Taylor, Padé and EOB models at 3.5PN order are within the numerical error (see Fig. 13). At high frequency, as already observed in Ref. [18], only the nonadiabatic $\mathrm{E}$ approximant has a $\mathrm{GW}$ frequency derivative that flattens out, as does the numerical result. The nonKeplerian E approximant at 3.5PN order is closest to the numerical data (see Fig. 14).

When estimating the derivative of center-of-mass energy $d E / d \varpi$, we expect the numerical result and adiabatic PN approximants to be close only in the range of frequencies over which the balance equation and the adiabatic approximation are valid. We find that this range of frequencies is $M \varpi \lesssim 0.08$ (see Fig. 15) for the 2.5PN T approximant and all the 3.5PN approximants. ${ }^{10}$ At higher frequency, the 3.5PN order nonadiabatic E approximants are closer to the numerical $d E / d \varpi$ than are the adiabatic approximants, and the non-Keplerian E approximant is the closest.

Applying a new matching procedure, we compared the numerical waveforms with TaylorT4, Padé, and EOB waveforms. We find that the accumulated phase difference from the numerical solution at $M \omega=0.1$ is -0.12 radians for the untuned 3.5PN P approximant [21,24,32], 0.50 radians for the untuned, original 3.5PN E approximant [22-24], and 0.45 radians for the untuned non-Keplerian [34] 3.5PN E approximant (see Fig. 16). Although those phase differences are larger than for 3.5PN TaylorT4 ( -0.04 radians), the phase differences for the $\mathrm{P}$ approx-

\footnotetext{
${ }^{10}$ It is not clear whether the failure of the adiabatic models is a result of the assumption of adiabaticity, or if the accuracy of those models would continue to improve if terms at order higher than 3.5PN were known.
} 
imants are less scattered as a function of PN order than are the phase differences for generic Taylor approximants.

The analyses of the flux, GW frequency derivative and (derivative of the) center-of-mass energy emphasize again the importance of including nonadiabatic effects during the last stages of inspiral [23]. Roughly, we can say that nonadiabatic effects are no longer negligible starting from a frequency $M \varpi \sim 0.08-0.12$, as can be seen in Figs. 9, 13, and 15. As seen in these figures, nonadiabatic E approximants can capture some of the relevant features of the late time evolution. We expect that by further improving these models by fitting higher-order PN coefficients to the numerical data, they will become excellent candidates for developing an analytic template bank of coalescing BBHs $[9,16,18,20,27]$.

In this paper, we started to explore the possibility of reducing the phase differences between numerical and $\mathrm{E}$ approximant waveforms by fitting the unknown parameters $a_{5}, \mathcal{F}_{8}$, and $v_{\text {pole }}$ (see Fig. 18). As a first step, for several E approximants we searched for a local minimal phase difference by varying $a_{5}, \mathcal{F}_{8}$, and $v_{\text {pole }}$. We found that we were able to reduce phase differences to below the numerical uncertainty. In a future work which will include merger and ringdown, we plan to determine the region of the parameter space $\left(a_{5}, \mathcal{F}_{8}, v_{\text {pole }}\right)$ in which the phase difference is within the numerical uncertainty of the simulation.

\section{ACKNOWLEDGMENTS}

We thank Emanuele Berti, Lee Lindblom, Etienne Racine, Bangalore Sathyaprakash, Saul Teukolsky, and Kip Thorne for informative discussions. We also thank Emanuele Berti and Eric Poisson for providing us the numerical data of the $\mathrm{GW}$ flux in the test-mass limit case. We thank Thibault Damour and Alessandro Nagar for clarifications on the "nontuned" EOB model used in Ref. [18]. A. B. and Y.P. acknowledge support from NSF Grant No. PHY-0603762, and A. B. also acknowledges support from the Alfred P. Sloan Foundation. M. B., L. K., A. M., H. P., and M. S. are supported in part by grants from the Sherman Fairchild Foundation to Caltech and Cornell, and from the Brinson Foundation to Caltech; by NSF Grants No. PHY-0601459, No PHY-0652995, No. DMS-0553302, and NASA Grant No. NNG05GG52G at Caltech; by NSF Grants No. PHY0652952, No. DMS-0553677, No. PHY-0652929, and NASA Grant No. NNG05GG51G at Cornell.

\section{APPENDIX: PADÉ APPROXIMANTS TO THE ENERGY FLUX IN THE TEST PARTICLE LIMIT}

In the test-mass limit case, the GW energy flux is known through 5.5PN order [46]. The explicit coefficients entering Eq. (19) for $i \geq 8$ and $\nu=0$ can be read from Eqs. (4.1) and (4.2) of Ref. [21].
In Fig. 19, we compare the normalized energy flux function [45] $F / F_{\text {Newt }}$ to the $\mathrm{T}$ and $\mathrm{P}$ approximants. To easily compare Fig. 19 with the other figures in the paper, we plot quantities as functions of the approximate $\mathrm{GW}$ frequency defined by $2 M \Omega$. As noticed in Ref. [21], the $\mathrm{P}$ approximants approach the numerical data more systematically. The differences between different PN orders are difficult to see in Fig. 19. To obtain a clearer view, Fig. 20 plots the differences between PN flux and numerical flux at four fixed frequencies. Figure 20 shows this somewhat better behavior of Padé; however, the Padé approximants show little improvement between PN orders 3.5 and 4.5, and at order 5 there occurs an extraneous pole. At frequency $2 M \Omega=0.04, \mathrm{P}$ approximants with order $\geq 2.5$ are
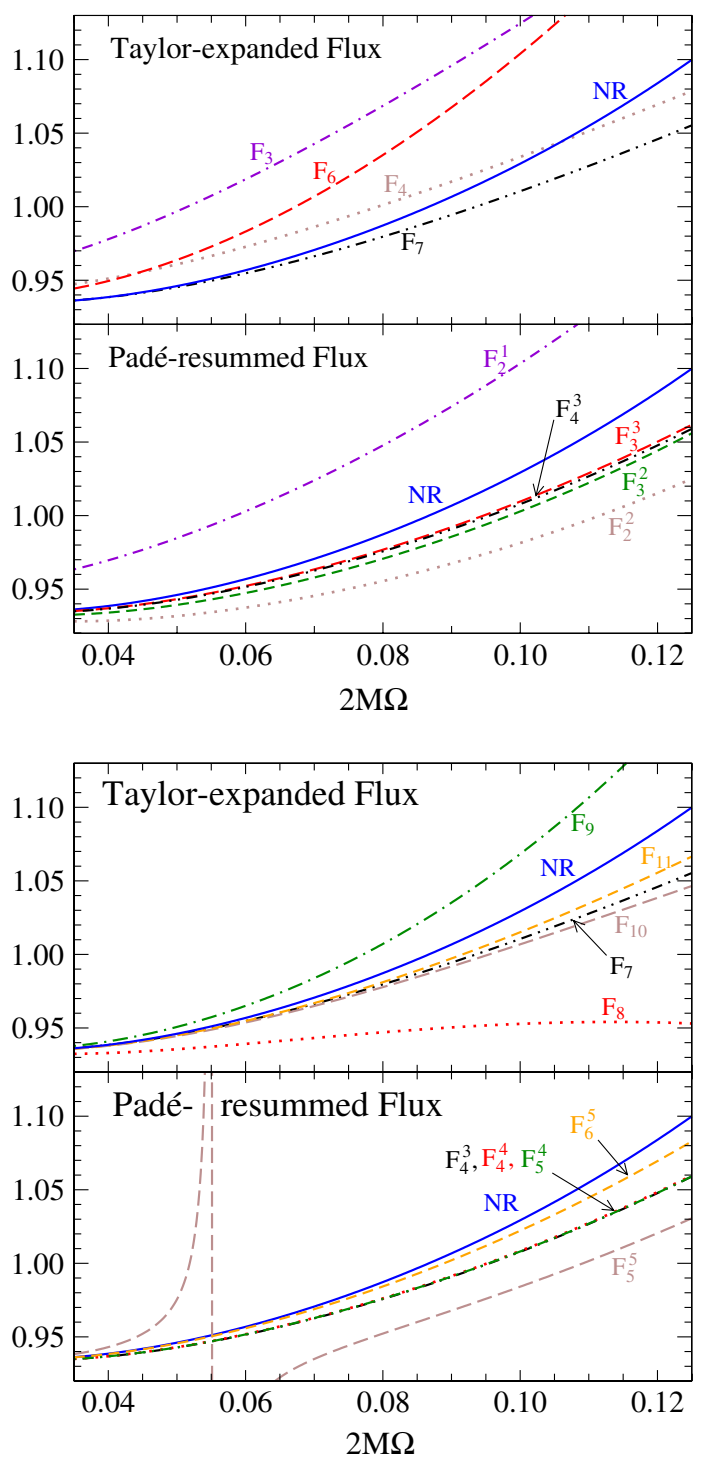

FIG. 19 (color online). Normalized energy flux $F / F_{\text {Newt }}$ versus GW frequency $2 \Omega$ in the test-mass limit. For notation see Table I and caption therein. For comparison, both panels also include the result of the numerical calculation of Poisson [45], labeled with NR. 


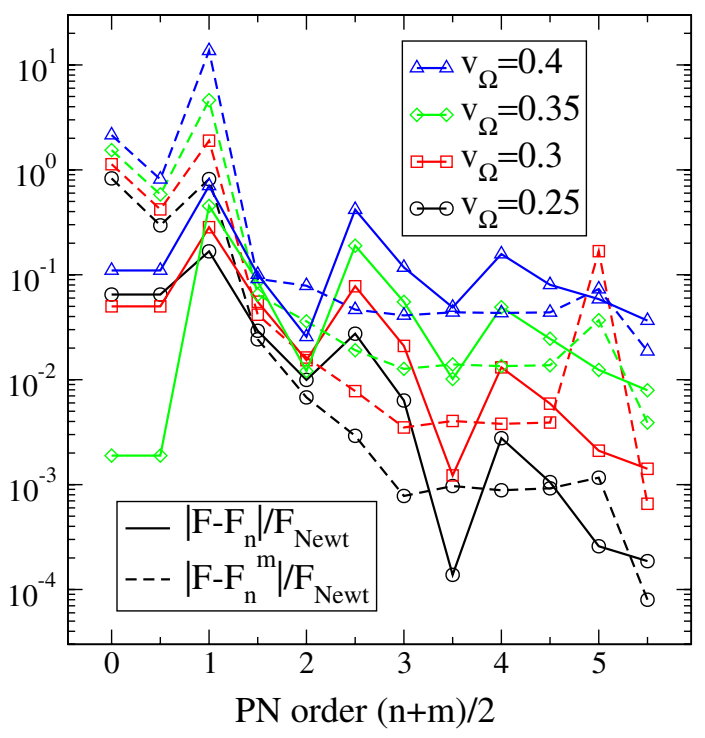

FIG. 20 (color online). Convergence of the PN approximants in the test-mass limit. Plotted are differences of $F / F_{\text {Newt }}$ from the numerical result. The $\mathrm{P}$ approximants do not converge faster than the Taylor series.

within $0.5 \%$ of the numerical data, as are $\mathrm{T}$ approximants with order $\geq 3.5$. Good agreement at low frequency is rather important because that is where the majority of the waveform phasing accumulates.

Table IV and Fig. 21 test the internal convergence of T and $\mathrm{P}$ approximants without referring to a numerical result. Table IV displays the flux at all known PN orders at select frequencies, with boldface highlighting the digits that have already converged. Although the Padé summation does not accelerate the convergence, the $\mathrm{P}$ approximant at 5.5PN order is closest to the numerical data (see Fig. 20).

Comparing Table IV with Table II, and Fig. 21 with Fig. 10 we observe that the $\mathrm{P}$ approximants converge

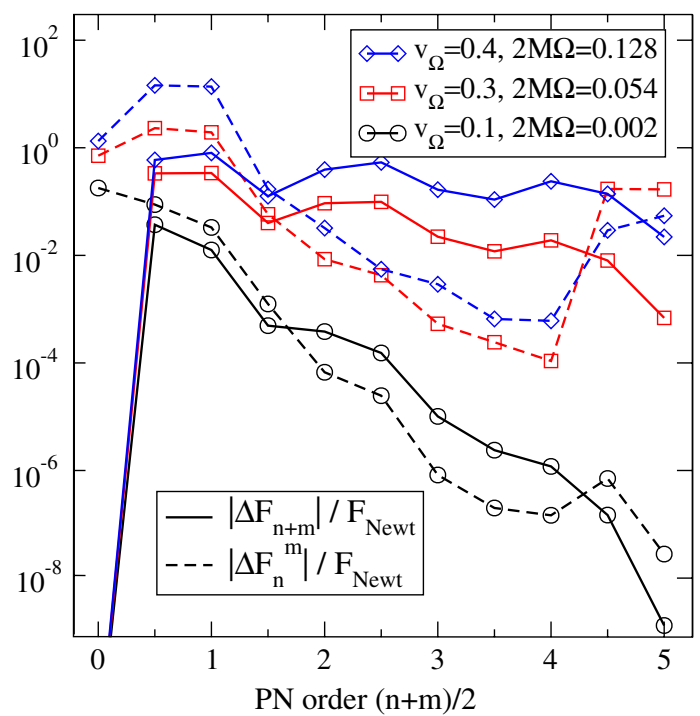

FIG. 21 (color online). Cauchy convergence test of $F / F_{\text {Newt }}$ in the test-mass limit for the $\mathrm{T}$ and $\mathrm{P}$ approximants. We plot $\Delta F_{n+m} \equiv F_{n+m+1}-F_{n+m}$, and $\Delta F_{n}^{m} \equiv F_{n+1}^{m}-F_{n}^{m}$ at three different frequencies. At high frequencies, the 4.5PN and 5PN Padé approximants are contaminated by the extraneous pole of the 5PN Padé series; for low frequencies $\left(v_{\Omega}=0.1\right)$, the pole is apparently irrelevant.

more systematically in the equal-mass case than in the test-mass limit. This is also evident by comparing Fig. 20 with Fig. 8: we see that $\mathrm{P}$ approximants at $3 \mathrm{PN}$ and 3.5PN orders are inside the numerical flux error whereas $\mathrm{T}$ approximants at all orders through $3.5 \mathrm{PN}$ are outside the numerical flux error bars even $\sim 25 \mathrm{GW}$ cycles before merger. However, as the Padé approximant does not converge faster, it is not immediately clear whether similar superior behavior of Padé can be expected for more generic binary black holes.

TABLE IV. Normalized energy flux $F / F_{\text {Newt }}$ in the test-mass limit for the T and P approximants at different PN orders and at three different frequencies. We use boldface to indicate the range of significant figures that do not change with increasing PN order.

\begin{tabular}{|c|c|c|c|c|c|c|}
\hline \multirow{2}{*}{$\begin{array}{l}\text { PN order } \\
(n+m) / 2 \\
\end{array}$} & \multicolumn{2}{|c|}{$v_{\Omega}=0.1 ; 2 M \Omega=0.002$} & \multicolumn{2}{|c|}{$v_{\Omega}=0.3 ; 2 M \Omega=0.054$} & \multicolumn{2}{|c|}{$v_{\Omega}=0.4,2 M \Omega=0.128$} \\
\hline & $F_{n+m} / F_{\text {Newt }}$ & $F_{n}^{m} / F_{\text {Newt }}$ & $F_{n+m} / F_{\text {Newt }}$ & $F_{n}^{m} / F_{\text {Newt }}$ & $F_{n+m} / F_{\text {Newt }}$ & $F_{n}^{m} / F_{\text {Newt }}$ \\
\hline 0.0 & 1.0000000000 & 1.20948977 & 1.0000 & 2.0817 & 1.000 & 3.255 \\
\hline 0.5 & 1.0000000000 & 1.03092783 & 1.0000 & 1.3699 & 1.000 & 1.923 \\
\hline 1.0 & $\mathbf{0 . 9 6 2 8 8 6 9 0 4 7}$ & $\mathbf{0 . 9 4 2 8 7 0 8 9}$ & 0.6660 & -0.9467 & 0.406 & -12.52 \\
\hline 1.5 & $\mathbf{0 . 9 7 5} 4532753$ & $\mathbf{0 . 9 7 5} 87569$ & 1.0053 & 0.9916 & 1.210 & 1.201 \\
\hline 2.0 & $\mathbf{0 . 9 7 4 9 6 0 4 2 9 2}$ & $\mathbf{0 . 9 7 4} 62770$ & 0.9653 & 0.9337 & 1.084 & 1.031 \\
\hline 2.5 & $\mathbf{0 . 9 7 4 5 7 7 5 0 0 9}$ & $\mathbf{0 . 9 7 4} 69475$ & 0.8723 & 0.9422 & 0.692 & 1.063 \\
\hline 3.0 & $\mathbf{0 . 9 7 4 7 3 0 7 7 5 7}$ & 0.97471937 & 0.9710 & 0.9465 & 1.227 & 1.069 \\
\hline 3.5 & 0.9747206248 & $\mathbf{0 . 9 7 4 7 1 8 5 4}$ & 0.9488 & 0.9460 & 1.061 & 1.066 \\
\hline 4.0 & $\mathbf{0 . 9 7 4 7 1 8 2 3 5 2}$ & 0.97471874 & 0.9369 & 0.9462 & 0.952 & 1.067 \\
\hline 4.5 & 0.9747194262 & $\mathbf{0 . 9 7 4 7 1 8 5 9}$ & 0.9559 & 0.9461 & 1.190 & 1.066 \\
\hline 5.0 & $\mathbf{0 . 9 7 4 7 1 9 2 7 7 6}$ & 0.97471930 & 0.9479 & 1.1178 & 1.051 & $\mathbf{1 . 0 3 7}$ \\
\hline 5.5 & 0.9747192763 & 0.97471928 & 0.9485 & 0.9493 & 1.073 & 1.091 \\
\hline
\end{tabular}


[1] B. C. Barish and R. Weiss, Phys. Today 52, 44 (1999).

[2] S. J. Waldman, Classical Quantum Gravity 23, S653 (2006).

[3] S. Hild, Classical Quantum Gravity 23, S643 (2006).

[4] F. Acernese, P. Amico, N. Arnaud, C. Arnault, D. Babusci, G. Ballardin, F. Barone, M. Barsuglia, F. Bellachia, J.L. Beney et al., Classical Quantum Gravity 19, 1421 (2002).

[5] F. Acernese, P. Amico, M. Alshourbagy, F. Antonucci, S. Aoudia, S. Avino, D. Babusci, G. Ballardin, F. Barone, L. Barsotti et al., Classical Quantum Gravity 23, S635 (2006).

[6] E. E. Flanagan and S. A. Hughes, Phys. Rev. D 57, 4535 (1998).

[7] T. Damour, B. R. Iyer, and B. S. Sathyaprakash, Phys. Rev. D 62, 084036 (2000).

[8] L. Blanchet, Living Rev. Relativity 9, 4 (2006).

[9] A. Buonanno, G. B. Cook, and F. Pretorius, Phys. Rev. D 75, 124018 (2007).

[10] J.G. Baker, J.R. van Meter, S. T. McWilliams, J. Centrella, and B.J. Kelly, Phys. Rev. Lett. 99, 181101 (2007).

[11] M. Hannam, S. Husa, J. A. González, U. Sperhake, and B. Brügmann, Phys. Rev. D 77, 044020 (2008).

[12] M. Boyle, D. A. Brown, L. E. Kidder, A. H. Mroué, H. P. Pfeiffer, M. A. Scheel, G. B. Cook, and S. A. Teukolsky, Phys. Rev. D 76, 124038 (2007).

[13] M. Hannam, S. Husa, B. Brügmann, and A. Gopakumar, arXiv:0712.3787 [Phys. Rev. D (to be published).

[14] A. Gopakumar, M. Hannam, S. Husa, and B. Brügmann, Phys. Rev. D 78, 064026 (2008).

[15] Y. Pan, A. Buonanno, J. G. Baker, J. Centrella, B. J. Kelly, S. T. McWilliams, F. Pretorius, and J. R. van Meter, Phys. Rev. D 77, 024014 (2008).

[16] A. Buonanno, Y. Pan, J. G. Baker, J. Centrella, B. J. Kelly, S. T. McWilliams, and J. R. van Meter, Phys. Rev. D 76, 104049 (2007).

[17] P. Ajith, S. Babak, Y. Chen, M. Hewitson, B. Krishnan, A. M. Sintes, J. T. Whelan, B. Brügmann, P. Diener, N. Dorband et al., Phys. Rev. D 77, 104017 (2008).

[18] T. Damour and A. Nagar, Phys. Rev. D 77, 024043 (2008).

[19] T. Damour, A. Nagar, E. N. Dorband, D. Pollney, and L. Rezzolla, Phys. Rev. D 77, 084017 (2008).

[20] T. Damour, A. Nagar, M. Hannam, S. Husa, and B. Brügmann, Phys. Rev. D 78, 044039 (2008).

[21] T. Damour, B. R. Iyer, and B. S. Sathyaprakash, Phys. Rev. D 57, 885 (1998).

[22] A. Buonanno and T. Damour, Phys. Rev. D 59, 084006 (1999).

[23] A. Buonanno and T. Damour, Phys. Rev. D 62, 064015 (2000).

[24] T. Damour, P. Jaranowski, and G. Schäfer, Phys. Rev. D 62, 084011 (2000).

[25] T. Damour, Phys. Rev. D 64, 124013 (2001).

[26] T. Damour, B. R. Iyer, P. Jaranowski, and B. S. Sathyaprakash, Phys. Rev. D 67, 064028 (2003).

[27] T. Damour and A. Nagar, Phys. Rev. D 76, 064028 (2007).

[28] H. P. Pfeiffer, D. A. Brown, L. E. Kidder, L. Lindblom, G. Lovelace, and M. A. Scheel, Classical Quantum Gravity 24, S59 (2007).
[29] E. Berti, V. Cardoso, J. A. Gonzalez, U. Sperhake, M. Hannam, S. Husa, and B. Brügmann, Phys. Rev. D 76, 064034 (2007).

[30] D. Pollney, C. Reisswig, L. Rezzolla, B. Szilágyi, M. Ansorg, B. Deris, P. Diener, E. N. Dorband, M. Koppitz, A. Nagar et al., Phys. Rev. D 76, 124002 (2007).

[31] J.D. Schnittman, A. Buonanno, J.R. van Meter, J. G. Baker, W. D. Boggs, J. Centrella, B. J. Kelly, and S. T. McWilliams, Phys. Rev. D 77, 044031 (2008).

[32] A. Buonanno, Y. Chen, and M. Vallisneri, Phys. Rev. D 67, 024016 (2003); 74, 029903(E) (2006).

[33] A. Buonanno, Y. Chen, and T. Damour, Phys. Rev. D 74, 104005 (2006).

[34] T. Damour and A. Gopakumar, Phys. Rev. D 73, 124006 (2006).

[35] P. Jaranowski and G. Schäfer, Phys. Rev. D 57, 7274 (1998); 63, 029902(E) (2000).

[36] V.C. de Andrade, L. Blanchet, and G. Faye, Classical Quantum Gravity 18, 753 (2001).

[37] T. Damour, P. Jaranowski, and G. Schäfer, Phys. Rev. D 62, 044024 (2000).

[38] L. Blanchet and G. Faye, Phys. Rev. D 63, 062005 (2001).

[39] T. Damour, P. Jaranowski, and G. Schäfer, Phys. Rev. D 63, 044021 (2001); 66, 029901(E) (2002).

[40] L. Blanchet, G. Faye, B. R. Iyer, and B. Joguet, Phys. Rev. D 65, 061501(R) (2002); 71, 129902(E) (2005).

[41] L. Blanchet, T. Damour, and G. Esposito-Farèse, Phys. Rev. D 69, 124007 (2004).

[42] L. Blanchet, T. Damour, G. Esposito-Farèse, and B. R. Iyer, Phys. Rev. D 71, 124004 (2005).

[43] L. Blanchet, T. Damour, G. Esposito-Farèse, and B. R. Iyer, Phys. Rev. Lett. 93, 091101 (2004).

[44] C. M. Bender and S. A. Orszag, Advanced Mathematical Methods for Scientists and Engineers (McGraw-Hill, New York, 1978).

[45] E. Poisson, Phys. Rev. D 52, 5719 (1995); 55, 7980 (1997).

[46] T. Tanaka, H. Tagoshi, and M. Sasaki, Prog. Theor. Phys. 96, 1087 (1996).

[47] T. Damour, B. R. Iyer, and B. S. Sathyaprakash, Phys. Rev. D 66, 027502 (2002); 72, 029901(E) (2005).

[48] LIGO Scientific Collaboration, LSC Algorithm Library software packages LAL, LALWRAPPER, and LALAPPS, http:// www.lsc-group.phys.uwm.edu/lal.

[49] E. Poisson, Phys. Rev. D 47, 1497 (1993).

[50] C. Cutler, L. S. Finn, E. Poisson, and G. J. Sussman, Phys. Rev. D 47, 1511 (1993).

[51] L. E. Kidder, Phys. Rev. D 77, 044016 (2008).

[52] K. Arun, L. Blanchet, B. Iyer, and M. Qusailah, Classical Quantum Gravity 21, 3771 (2004); 22, 3115(E) (2005).

[53] L. Blanchet, G. Faye, B. R. Iyer, and S. Sinha, Classical Quantum Gravity 25, 165003 (2008).

[54] J. G. Baker, S.T. McWilliams, J.R. van Meter, J. Centrella, D.-I. Choi, B. J. Kelly, and M. Koppitz, Phys. Rev. D 75, 124024 (2007).

[55] G. Lovelace, R. Owen, H. P. Pfeiffer, and T. Chu, Phys. Rev. D 78, 084017 (2008).

[56] N. Yunes and E. Berti, Phys. Rev. D 77, 124006 (2008). 